\title{
Characteristics of global strong earthquakes and their implications for the present-day stress pattern
}

\author{
Ju Wei ${ }^{1,2,3, *}$ (i), Sun Weifeng ${ }^{2}$ and Luo Jinhui ${ }^{2,3}$ \\ ${ }^{1}$ Key Laboratory of Coal-based $\mathrm{CO}_{2}$ Capture and Geological Storage, Xuzhou 221008, China. \\ ${ }^{2}$ School of Resources and Geosciences, China University of Mining and Technology, Xuzhou 221116, China. \\ ${ }^{3}$ Key Laboratory of Coalbed Methane Resources and Reservoir Formation Process, Ministry of Education, \\ China University of Mining and Technology, Xuzhou 221008, China. \\ *Corresponding author.e-mail: wju@cumt.edu.cn
}

MS received 15 June 2016; revised 4 April 2017; accepted 10 May 2017; published online 6 October 2017

Earthquakes occurred on the surface of the Earth contain comprehensive and abundant geodynamic connotations, and can serve as important sources for describing the present-day stress field and regime. An important advantage of the earthquake focal mechanism solution is the ability to obtain the stress pattern information at depth in the lithosphere. During the past several decades, an increasing number of focal mechanisms were available for estimating the present-day stress field and regime. In the present study, altogether 553 focal mechanism data ranging from the year 1976 to 2017 with $M w \geq 7.0$ were compiled in the Global/Harvard centroid moment tensor (CMT) catalogue, the characteristics of global strong earthquakes and the present-day stress pattern were analyzed based on these data. The majority of global strong earthquakes are located around the plate boundaries, shallow-focus, and thrust faulting (TF) regime. We grouped 518 of them into 12 regions (Boxes) based on their geographical proximity and tectonic setting. For each box, the present-day stress field and regime were obtained by formal stress inversion. The results indicated that the maximum horizontal principal stress direction was $\sim \mathrm{N}-\mathrm{S}$ trending in western North America continent and southwestern Indonesia, $\sim \mathrm{NNE}-\mathrm{SSW}$-trending in western Middle America and central Asia, $\sim \mathrm{NE}-\mathrm{SW}$ in southeastern South America continent and northeastern Australia, NEE-SWW-trending in western South America continent and southeastern Asia, $\sim \mathrm{E}-\mathrm{W}$-trending in southeastern Australia, and $\sim \mathrm{NW}-\mathrm{SE}$-trending in eastern Asia. The results can provide additional constraints to the driving forces and geodynamic models, allowing them to explain the current plate interactions and crustal tectonic complexities better.

Keywords. Global strong earthquake; formal stress inversion; present-day stress pattern; focal mechanism solution.

\section{Introduction}

Generally, the lithospheric plates move towards and against each other with different velocities in different directions, and relative movements of these plates along the plate boundaries will induce earthquakes. The gliding process between plates produces great but variable stress (Frisch et al. 2011). In recent years, the world has stepped into a natural disaster-prone period, and frequent natural disasters such as earthquakes pose a great threat to life and property safety around the world. 
Tectonic stress is one of the fundamental datasets in Earth sciences compared to topography, gravity, heat flow and others (Sperner et al. 2003), which is released in catastrophic earthquakes (Fuchs and Muller 2001; Sperner et al. 2003). Knowledge of stress field in the hydrocarbon industry can be used to foresee borehole stability problems, optimize reservoir management through tectonic modeling in combination with other datasets, and predict tectonic fractures (Ding et al. 1998; Sperner et al. 2003; Smart et al. 2009; Ju et al. 2013b, 2014b, 2015; Ju and Sun 2016). Thus, investigation into the crustal tectonic stress is a key point in Earth geotectonic studies (Zoback 1992; Hou et al. 2006; Lunina and Gladkov 2007; Heidbach et al. 2010; Ju et al. 2012, 2013a, 2014a; Hussein et al. 2013).

Generally, formal inversion of focal mechanism data can reveal the present-day stress field and geodynamic regime (Zoback 1992; Fuchs and Muller 2001; Cortes and Angelier 2005; Delvaux and Barth 2010; Naimi-Ghassabian et al. 2015). Focal mechanism solutions are highly valuable as they can offer reliable stress information at different depths in the lithosphere (Tingay et al. 2010; Hussein et al. 2013). Observation of the present-day stress field and regime in the crust represents an important task for understanding the driving forces for the current deformation and the style of deformation itself (Hussein et al. 2013).

The purpose of this study is to analyze the characteristics of global strong earthquakes, estimate the orientations of the principal stress axes, and deduce the present-day stress pattern in the world. In the present study, it is considered that the strong earthquake is defined when its magnitude $(M w)$ is equal to or larger than 7.0. During 19762017, altogether 553 focal mechanism data with $M w \geq 7.0$ were compiled from the Global/Harvard CMT catalogue, and the characteristics of global strong earthquakes were analyzed. Further, 518 of them were grouped into 12 regions (Boxes) based on their tectonic setting and geographical proximity. Formal inversions were performed to determine the present-day stress pattern globally based on these groups.

\section{Data compilation}

The Global CMT Project, formerly Harvard CMT, regularly determines focal mechanisms by moment tensor inversion of both long period body- and surface-waves to fit seismic waveforms calculated for a reference earth model (Dziewonski et al. 1981, 1987; Zoback 1992). In this study, we combined 553 CMT solutions with $M w \geq 7.0$ from the year 1976 to 2017 (table 1) to perform stress inversions for separate regions (figure 1).

Based on the compiled database, the individual focal mechanism was analyzed. The horizontal stress axes $S_{H \max }$ and $S_{H \text { min }}$, according to Lund and Townend (2007) and stress regime based on the World Stress Map (WSM) standard were calculated (table 1 and figure 2; NF: normal faulting, SS: strike-slip faulting, TF: thrust faulting, NS: intermediate between NF and SS, TS: intermediate between SS and TF, and UF: unknown or oblique faulting).

\section{Characteristics of global strong earthquakes}

\subsection{Temporal characteristics}

Based on the complied database (table 1), the temporal characteristics of global strong earthquakes are analyzed. During 1976-2008, the number of strong earthquakes is generally less than 15 each year, whereas it increases greatly during 20092017 with a maximum of 23 in the year 2010 (figure 3a). The results indicate that the world has stepped into a natural disaster-prone period with frequent occurrence of strong earthquakes (figure $3 \mathrm{a}$ ) in recent years. In addition, the earthquake magnitude becomes larger as well with several superstrong earthquakes (e.g., $M w=9.1,20110311$, 05:47:33; figure $3 \mathrm{~b}$ ).

\subsection{Spatial characteristics}

Global strong earthquakes are not distributed randomly, but concentrated on a few worldwide seismic belts. Based on the distribution of global strong earthquakes, they are primarily located around plate boundaries (figure 1). Relative movements among plates will accumulate great energy, leading to strong earthquakes. In the latitudinal direction, global strong earthquakes are located between $70^{\circ} \mathrm{S}$ and $70^{\circ} \mathrm{N}$ with regions between $30^{\circ} \mathrm{S}$ and $60^{\circ} \mathrm{N}$ accounting for about $91.36 \%$ (figure $4 \mathrm{a}$ ). In the longitudinal direction, global strong earthquakes are primarily located in three zones: $180^{\circ}$ $170^{\circ} \mathrm{W}, 80^{\circ}-70^{\circ} \mathrm{W}$ and $120^{\circ}-170^{\circ} \mathrm{E}$ (figure $4 \mathrm{~b}$ ). 
Table 1. Complied database of global focal mechanisms (1976 - 2017).

\begin{tabular}{|c|c|c|c|c|c|c|c|c|c|c|}
\hline \multirow[b]{2}{*}{ Time } & \multicolumn{3}{|c|}{ Location } & \multicolumn{4}{|c|}{ Focal mechanism data } & \multicolumn{2}{|c|}{$\mathrm{SH}$} & \multirow{2}{*}{$\begin{array}{l}\text { Stress } \\
\text { Regime }\end{array}$} \\
\hline & Lat. & Long. & Depth & $M w$ & Strike & Dip & Slip & Max & Min & \\
\hline 19760101-012953 & -29.25 & -176.96 & 047.8 & 7.3 & 018 & 60 & 088 & 110 & 020 & $\mathrm{TF}$ \\
\hline 19760114-155608 & -29.69 & -177.04 & 046.7 & 7.8 & 015 & 64 & 088 & 108 & 018 & $\mathrm{TF}$ \\
\hline 19760114-164745 & -28.72 & -176.75 & 017.7 & 7.9 & 028 & 80 & 093 & 108 & 018 & UF \\
\hline 19760121-100534 & 44.58 & 149.49 & 026.5 & 7.2 & 030 & 76 & 083 & 134 & 044 & $\mathrm{TF}$ \\
\hline 19760204-090107 & 15.14 & -089.78 & 016.3 & 7.5 & 347 & 80 & -162 & 031 & 121 & SS \\
\hline 19760324-044616 & -29.99 & -177.51 & 054.1 & 7.0 & 011 & 57 & 081 & 109 & 019 & $\mathrm{TF}$ \\
\hline $19760505-045203$ & -29.84 & -177.43 & 041.8 & 7.0 & 013 & 57 & 080 & 112 & 022 & $\mathrm{TF}$ \\
\hline $19760603-164453$ & -04.75 & 153.47 & 085.9 & 7.1 & 331 & 59 & 094 & 057 & 147 & $\mathrm{TF}$ \\
\hline $19760620-205324$ & 03.18 & 096.24 & 019.1 & 7.0 & 147 & 62 & 085 & 063 & 153 & $\mathrm{TF}$ \\
\hline $19760625-191813$ & -04.66 & 140.29 & 015.0 & 7.1 & 113 & 67 & 034 & 061 & 151 & UF \\
\hline 19760711-204105 & 07.32 & -078.11 & 015.0 & 7.3 & 176 & 72 & 024 & 127 & 037 & SS \\
\hline $19760727-194211$ & 39.52 & 118.03 & 015.0 & 7.6 & 126 & 79 & -049 & 087 & 177 & UF \\
\hline $19760728-104546$ & 39.75 & 118.78 & 015.0 & 7.0 & 279 & 49 & -071 & 086 & 176 & NF \\
\hline 19760816-161159 & 07.07 & 123.75 & 033.0 & 8.0 & 158 & 55 & 089 & 069 & 159 & $\mathrm{TF}$ \\
\hline $19760817-041940$ & 07.14 & 123.01 & 015.0 & 7.1 & 120 & 83 & -026 & 077 & 167 & SS \\
\hline $19761124-122225$ & 38.88 & 043.96 & 015.0 & 7.0 & 110 & 81 & 167 & 156 & 066 & SS \\
\hline 19761130-004013 & -20.54 & -068.51 & 133.7 & 7.5 & 342 & 64 & -099 & 172 & 082 & NF \\
\hline 19770304-192211 & 45.23 & 026.17 & 083.6 & 7.5 & 235 & 62 & 092 & 143 & 053 & $\mathrm{TF}$ \\
\hline $19770318-214407$ & 16.38 & 122.59 & 035.0 & 7.2 & 007 & 65 & 081 & 108 & 018 & $\mathrm{TF}$ \\
\hline $19770402-071538$ & -16.77 & -171.91 & 050.3 & 7.3 & 190 & 62 & 122 & 073 & 163 & $\mathrm{TF}$ \\
\hline $19770420-234304$ & -09.20 & 159.20 & 016.3 & 7.2 & 131 & 67 & 102 & 027 & 117 & $\mathrm{TF}$ \\
\hline $19770420-234916$ & -09.33 & 159.79 & 016.4 & 7.1 & 122 & 72 & 077 & 050 & 140 & $\mathrm{TF}$ \\
\hline $19770421-042423$ & -10.22 & 160.59 & 040.4 & 7.3 & 159 & 55 & 097 & 063 & 153 & $\mathrm{TF}$ \\
\hline $19770622-120922$ & -22.86 & -174.91 & 061.3 & 8.0 & 197 & 79 & -089 & 014 & 104 & NF \\
\hline 19770729-111549 & -08.16 & 155.92 & 024.3 & 7.1 & 272 & 80 & 034 & 043 & 133 & SS \\
\hline 19770819-060933 & -11.14 & 118.23 & 023.3 & 8.3 & 061 & 67 & -098 & 071 & 161 & NF \\
\hline 19770826-195005 & -59.60 & -019.70 & 030.2 & 7.2 & 274 & 89 & 156 & 140 & 050 & SS \\
\hline $19770827-071234$ & -07.92 & 125.72 & 032.3 & 7.0 & 038 & 49 & 068 & 144 & 054 & $\mathrm{TF}$ \\
\hline 19771010-115403 & -26.10 & -174.88 & 023.4 & 7.3 & 170 & 55 & -107 & 004 & 094 & NF \\
\hline $19771017-172657$ & -27.93 & 173.88 & 017.4 & 7.0 & 348 & 90 & -145 & 033 & 123 & UF \\
\hline $19771123-092648$ & -31.22 & -067.69 & 020.8 & 7.4 & 004 & 46 & 090 & 094 & 004 & $\mathrm{TF}$ \\
\hline 19780209-213527 & -30.60 & -177.02 & 023.1 & 7.0 & 026 & 61 & 095 & 110 & 020 & $\mathrm{TF}$ \\
\hline $19780307-024855$ & 31.90 & 137.44 & 434.4 & 7.1 & 153 & 76 & 077 & 086 & 176 & $\mathrm{TF}$ \\
\hline $19780323-003115$ & 43.81 & 149.23 & 041.5 & 7.0 & 038 & 70 & 087 & 132 & 042 & $\mathrm{TF}$ \\
\hline $19780323-031545$ & 44.12 & 149.27 & 028.3 & 7.6 & 042 & 79 & 090 & 132 & 042 & $\mathrm{TF}$ \\
\hline 19780324-194811 & 44.20 & 148.98 & 030.7 & 7.5 & 040 & 72 & 089 & 132 & 042 & $\mathrm{TF}$ \\
\hline $19780612-081443$ & 38.02 & 142.07 & 037.7 & 7.6 & 036 & 78 & 097 & 110 & 020 & $\mathrm{TF}$ \\
\hline $19780723-144253$ & 22.01 & 121.60 & 028.9 & 7.2 & 005 & 61 & 069 & 114 & 024 & $\mathrm{TF}$ \\
\hline 19780916-153614 & 33.37 & 057.02 & 011.0 & 7.3 & 128 & 59 & 080 & 047 & 137 & $\mathrm{TF}$ \\
\hline $19781104-221938$ & -11.16 & 161.82 & 033.3 & 7.0 & 161 & 73 & 119 & 040 & 130 & $\mathrm{TF}$ \\
\hline $19781105-220213$ & -10.94 & 162.09 & 040.0 & 7.0 & 154 & 73 & 107 & 041 & 131 & $\mathrm{TF}$ \\
\hline 19781129-195303 & 16.22 & -096.56 & 016.1 & 7.7 & 127 & 84 & 094 & 020 & 110 & UF \\
\hline 19781206-140236 & 44.74 & 145.82 & 181.0 & 7.8 & 330 & 83 & -033 & 107 & 017 & SS \\
\hline $19781223-112325$ & 23.19 & 122.63 & 020.7 & 7.0 & 343 & 72 & 039 & 111 & 021 & UF \\
\hline $19790216-100858$ & -16.88 & -072.48 & 034.3 & 7.1 & 305 & 84 & 104 & 001 & 091 & UF \\
\hline $19790228-212738$ & 60.50 & -141.39 & 018.8 & 7.4 & 084 & 77 & 089 & 176 & 086 & $\mathrm{TF}$ \\
\hline 19790314-110731 & 17.78 & -101.37 & 026.7 & 7.4 & 106 & 76 & 085 & 026 & 116 & $\mathrm{TF}$ \\
\hline $19790410-014225$ & 03.13 & 127.32 & 040.6 & 7.0 & 358 & 75 & 104 & 066 & 156 & $\mathrm{TF}$ \\
\hline $19790501-130355$ & -21.27 & 169.97 & 073.4 & 7.4 & 281 & 83 & -050 & 060 & 150 & UF \\
\hline 19790724-193128 & -11.26 & 107.68 & 020.0 & 7.0 & 334 & 90 & -044 & 109 & 019 & UF \\
\hline $19790912-051800$ & -01.78 & 136.06 & 016.3 & 7.5 & 008 & 85 & 161 & 054 & 144 & SS \\
\hline $19791012-102530$ & -46.54 & 165.77 & 020.3 & 7.3 & 152 & 80 & 079 & 085 & 175 & $\mathrm{TF}$ \\
\hline
\end{tabular}


Table 1. (Continued.)

\begin{tabular}{|c|c|c|c|c|c|c|c|c|c|c|}
\hline \multirow[b]{2}{*}{ Time } & \multicolumn{3}{|c|}{ Location } & \multicolumn{4}{|c|}{ Focal mechanism data } & \multicolumn{2}{|c|}{$\mathrm{SH}$} & \multirow{2}{*}{$\begin{array}{l}\text { Stress } \\
\text { Regime }\end{array}$} \\
\hline & Lat. & Long. & Depth & $M w$ & Strike & Dip & Slip & Max & Min & \\
\hline $19791017-054313$ & 18.54 & 145.30 & 583.8 & 7.0 & 140 & 55 & -135 & 170 & 080 & NF \\
\hline 19791023-095115 & -10.58 & 161.35 & 031.3 & 7.0 & 158 & 62 & 097 & 061 & 151 & $\mathrm{TF}$ \\
\hline $19791123-234026$ & 05.18 & -075.58 & 108.6 & 7.2 & 035 & 79 & -051 & 177 & 087 & UF \\
\hline $19791127-171044$ & 34.45 & 059.58 & 025.0 & 7.0 & 358 & 73 & -156 & 040 & 130 & SS \\
\hline $19791212-080007$ & 02.32 & -078.81 & 019.7 & 8.1 & 181 & 76 & 083 & 105 & 015 & $\mathrm{TF}$ \\
\hline $19800223-055112$ & 43.21 & 146.91 & 033.6 & 7.1 & 034 & 75 & 090 & 124 & 034 & $\mathrm{TF}$ \\
\hline $19800308-221220$ & -22.86 & 171.65 & 026.8 & 7.2 & 089 & 75 & 081 & 015 & 105 & $\mathrm{TF}$ \\
\hline $19800413-180453$ & -23.74 & -176.70 & 166.2 & 7.6 & 30 & 79 & -067 & 177 & 087 & UF \\
\hline $19800708-231941$ & -12.92 & 166.21 & 043.6 & 7.5 & 353 & 49 & 100 & 076 & 166 & $\mathrm{TF}$ \\
\hline $19800716-195653$ & -04.68 & 143.18 & 081.9 & 7.3 & 085 & 68 & 086 & 00 & 090 & $\mathrm{TF}$ \\
\hline $19800717-194303$ & -12.44 & 165.94 & 034.0 & 7.7 & 158 & 60 & 083 & 075 & 165 & $\mathrm{TF}$ \\
\hline $19801010-122534$ & 36.25 & 001.36 & 010.1 & 7.1 & 050 & 61 & 081 & 150 & 060 & $\mathrm{TF}$ \\
\hline 19801024-145348 & 18.14 & -097.86 & 063.4 & 7.1 & 105 & 66 & -101 & 118 & 028 & NF \\
\hline $19801025-070026$ & -22.16 & 169.83 & 011.0 & 7.1 & 124 & 65 & 081 & 045 & 135 & $\mathrm{TF}$ \\
\hline $19801025-110025$ & -21.76 & 169.84 & 028.8 & 7.4 & 136 & 68 & 085 & 053 & 143 & $\mathrm{TF}$ \\
\hline $19801108-102746$ & 41.14 & -124.36 & 015.0 & 7.3 & 051 & 89 & 27 & 005 & 095 & SS \\
\hline $19810118-181736$ & 38.15 & 143.04 & 020.0 & 7.0 & 027 & 76 & 095 & 107 & 017 & $\mathrm{TF}$ \\
\hline $19810130-085250$ & 51.52 & 176.10 & 020.0 & 7.0 & 070 & 70 & 081 & 172 & 082 & $\mathrm{TF}$ \\
\hline $19810525-052538$ & -48.94 & 164.39 & 033.3 & 7.6 & 033 & 85 & -173 & 078 & 168 & SS \\
\hline $19810706-030852$ & -22.31 & 170.90 & 058.3 & 7.5 & 254 & 90 & -060 & 30 & 120 & UF \\
\hline $19810715-075925$ & -17.34 & 167.27 & 030.0 & 7.1 & 166 & 63 & 094 & 072 & 162 & $\mathrm{TF}$ \\
\hline $19810728-172244$ & 30.03 & 057.58 & 015.2 & 7.2 & 300 & 79 & 084 & 045 & 135 & $\mathrm{TF}$ \\
\hline 19810901-092947 & -15.02 & -173.16 & 020.0 & 7.5 & 273 & 55 & -103 & 104 & 014 & NF \\
\hline $19811016-032548$ & -33.48 & -073.15 & 040.4 & 7.1 & 006 & 56 & 086 & 100 & 010 & $\mathrm{TF}$ \\
\hline $19811025-032234$ & 18.28 & -102.00 & 031.8 & 7.2 & 115 & 70 & 093 & 021 & 111 & $\mathrm{TF}$ \\
\hline $19811226-170543$ & -29.86 & -177.75 & 030.5 & 7.0 & 007 & 63 & 083 & 104 & 014 & $\mathrm{TF}$ \\
\hline $19820103-141004$ & -00.96 & -021.83 & 010.0 & 7.1 & 351 & 81 & 031 & 124 & 034 & SS \\
\hline 19820111-061015 & 13.91 & 124.35 & 017.3 & 7.1 & 351 & 80 & 098 & 062 & 152 & $\mathrm{TF}$ \\
\hline $19820507-053843$ & -60.96 & -020.62 & 010.0 & 7.0 & 358 & 90 & -143 & 043 & 133 & UF \\
\hline 19820619-062202 & 12.65 & -088.97 & 051.9 & 7.3 & 299 & 66 & -083 & 111 & 021 & NF \\
\hline $19820622-41853$ & 07.28 & 125.99 & 473.4 & 7.4 & 232 & 65 & -056 & 023 & 113 & NF \\
\hline $19820630-015744$ & 44.59 & 151.18 & 026.5 & 7.1 & 221 & 89 & -118 & 084 & 174 & UF \\
\hline $19820707-104315$ & -51.18 & 160.41 & 010.0 & 7.2 & 334 & 88 & -028 & 110 & 020 & SS \\
\hline $19820723-142410$ & 35.98 & 141.91 & 027.0 & 7.0 & 027 & 76 & 091 & 115 & 025 & TF \\
\hline $19821219-174422$ & -24.31 & -175.10 & 029.2 & 7.5 & 006 & 68 & 086 & 101 & 011 & $\mathrm{TF}$ \\
\hline $19830126-160229$ & -30.52 & -179.21 & 224.1 & 7.0 & 086 & 83 & -092 & 094 & 004 & UF \\
\hline 19830318-090606 & -04.86 & 153.34 & 069.9 & 7.7 & 308 & 49 & 060 & 059 & 149 & $\mathrm{TF}$ \\
\hline 19830403-025021 & 08.85 & -083.25 & 028.0 & 7.4 & 108 & 67 & 081 & 029 & 119 & TF \\
\hline 19830404-025144 & 05.72 & 094.65 & 071.6 & 7.0 & 078 & 53 & 127 & 142 & 052 & $\mathrm{TF}$ \\
\hline $19830412-120800$ & -04.92 & -078.07 & 111.1 & 7.0 & 153 & 55 & -093 & 155 & 065 & NF \\
\hline $19830526-030018$ & 40.44 & 138.87 & 012.6 & 7.7 & 200 & 63 & 092 & 108 & 018 & $\mathrm{TF}$ \\
\hline $19830817-105606$ & 55.30 & 161.75 & 077.2 & 7.0 & 073 & 55 & 114 & 144 & 054 & $\mathrm{TF}$ \\
\hline $19831004-185238$ & -26.01 & -070.56 & 038.7 & 7.6 & 168 & 71 & 083 & 089 & 179 & $\mathrm{TF}$ \\
\hline $19831022-042149$ & -60.59 & -024.19 & 010.1 & 7.0 & 042 & 56 & -095 & 047 & 137 & NF \\
\hline $19831124-053043$ & -07.44 & 128.32 & 157.1 & 7.4 & 291 & 57 & 113 & 002 & 092 & $\mathrm{TF}$ \\
\hline $19831130-174629$ & -06.35 & 071.75 & 010.0 & 7.7 & 069 & 63 & -113 & 091 & 001 & NF \\
\hline 19831202-030911 & 13.86 & -092.20 & 031.0 & 7.0 & 118 & 71 & 090 & 028 & 118 & $\mathrm{TF}$ \\
\hline $19831230-235300$ & 37.17 & 070.41 & 212.2 & 7.4 & 103 & 72 & 093 & 009 & 099 & $\mathrm{TF}$ \\
\hline 19840101-090347 & 33.38 & 136.81 & 383.6 & 7.1 & 162 & 75 & 073 & 097 & 007 & $\mathrm{TF}$ \\
\hline $19840207-213336$ & -09.81 & 160.42 & 021.9 & 7.5 & 182 & 72 & 124 & 059 & 149 & UF \\
\hline $19840305-033358$ & 08.26 & 123.86 & 644.1 & 7.2 & 038 & 58 & -076 & 026 & 116 & NF \\
\hline $19840306-021731$ & 29.60 & 139.11 & 446.0 & 7.4 & 332 & 88 & -070 & 110 & 020 & UF \\
\hline
\end{tabular}


Table 1. (Continued.)

\begin{tabular}{|c|c|c|c|c|c|c|c|c|c|c|}
\hline \multirow[b]{2}{*}{ Time } & \multicolumn{3}{|c|}{ Location } & \multicolumn{4}{|c|}{ Focal mechanism data } & \multicolumn{2}{|c|}{$\mathrm{SH}$} & \multirow{2}{*}{$\begin{array}{l}\text { Stress } \\
\text { Regime }\end{array}$} \\
\hline & Lat. & Long. & Depth & $M w$ & Strike & Dip & Slip & Max & Min & \\
\hline $19840319-202848$ & 40.59 & 063.24 & 015.0 & 7.0 & 041 & 69 & 091 & 129 & 039 & $\mathrm{TF}$ \\
\hline 19840324-094416 & 44.17 & 148.62 & 030.6 & 7.1 & 030 & 74 & 085 & 129 & 039 & $\mathrm{TF}$ \\
\hline 19840530-074955 & -05.02 & 151.64 & 157.0 & 7.1 & 038 & 64 & 077 & 142 & 052 & TF \\
\hline 19840806-120207 & -00.29 & 122.38 & 252.8 & 7.4 & 230 & 75 & -120 & 083 & 173 & UF \\
\hline $19841015-102118$ & 15.81 & -173.40 & 119.7 & 7.1 & 161 & 89 & -105 & 024 & 114 & UF \\
\hline 19841101-044859 & 08.36 & -038.82 & 010.0 & 7.0 & 183 & 88 & -017 & 138 & 048 & SS \\
\hline $19841115-024630$ & -22.10 & 170.67 & 098.6 & 7.0 & 093 & 86 & 063 & 044 & 134 & UF \\
\hline 19841117-064939 & -00.23 & 097.84 & 024.7 & 7.1 & 128 & 81 & 086 & 051 & 141 & UF \\
\hline $19841120-081524$ & 05.02 & 125.56 & 180.7 & 7.5 & 335 & 66 & -097 & 163 & 073 & NF \\
\hline $19850303-224740$ & -33.92 & -071.71 & 040.7 & 7.9 & 169 & 66 & 081 & 090 & 00 & $\mathrm{TF}$ \\
\hline 19850304-003253 & -33.06 & -071.79 & 033.0 & 7.3 & 346 & 77 & 060 & 110 & 020 & UF \\
\hline 19850409-015708 & -34.26 & -071.86 & 046.6 & 7.1 & 170 & 69 & 086 & 085 & 175 & $\mathrm{TF}$ \\
\hline $19850510-153601$ & -05.68 & 150.97 & 025.4 & 7.2 & 097 & 77 & -023 & 055 & 145 & SS \\
\hline $19850703-043707$ & -04.28 & 152.58 & 031.4 & 7.2 & 329 & 55 & 078 & 068 & 158 & $\mathrm{TF}$ \\
\hline 19850729-075458 & 36.33 & 070.96 & 098.5 & 7.4 & 254 & 54 & 104 & 152 & 062 & TF \\
\hline $19850919-131825$ & 17.91 & -101.99 & 021.3 & 8.0 & 106 & 73 & 085 & 024 & 114 & TF \\
\hline 19850921-013732 & 17.57 & 101.42 & 020.8 & 7.5 & 121 & 73 & 091 & 029 & 119 & $\mathrm{TF}$ \\
\hline $19851117-094036$ & -01.63 & 134.71 & 013.3 & 7.1 & 271 & 85 & 026 & 045 & 135 & SS \\
\hline $19851128-035008$ & -13.76 & 166.08 & 044.4 & 7.0 & 167 & 78 & 158 & 035 & 125 & SS \\
\hline $19851221-011333$ & -13.89 & 166.57 & 045.5 & 7.1 & 348 & 46 & 092 & 077 & 167 & TF \\
\hline $19860115-201748$ & -21.26 & 169.91 & 121.0 & 7.0 & 122 & 83 & 063 & 071 & 161 & UF \\
\hline $19860507-224745$ & 51.33 & -175.43 & 031.3 & 7.9 & 072 & 68 & 092 & 160 & 070 & $\mathrm{TF}$ \\
\hline $19860526-190625$ & -20.24 & 179.03 & 567.5 & 7.1 & 103 & 89 & 069 & 056 & 146 & UF \\
\hline $19860616-104836$ & -21.86 & -178.87 & 564.8 & 7.0 & 040 & 72 & -097 & 051 & 141 & NF \\
\hline $19860624-031143$ & -04.42 & 143.64 & 099.7 & 7.2 & 115 & 73 & 082 & 039 & 129 & $\mathrm{TF}$ \\
\hline 19860814-193929 & 02.08 & 126.89 & 020.2 & 7.5 & 224 & 81 & 104 & 106 & 016 & UF \\
\hline $19860830-212851$ & 45.76 & 026.53 & 132.7 & 7.2 & 240 & 72 & 097 & 138 & 048 & $\mathrm{TF}$ \\
\hline $19861020-064623$ & -27.93 & -176.07 & 050.4 & 7.7 & 013 & 71 & 036 & 142 & 052 & SS \\
\hline 19861030-012905 & -21.68 & -176.45 & 196.3 & 7.1 & 020 & 85 & -091 & 026 & 116 & UF \\
\hline 19861114-212018 & 23.97 & 121.85 & 033.2 & 7.3 & 033 & 57 & 092 & 121 & 031 & $\mathrm{TF}$ \\
\hline $19870208-183414$ & -05.90 & 147.72 & 015.0 & 7.3 & 352 & 87 & 173 & 037 & 127 & SS \\
\hline $19870305-091721$ & -24.38 & -070.93 & 041.9 & 7.5 & 175 & 68 & 083 & 094 & 004 & $\mathrm{TF}$ \\
\hline 19870306-041055 & -00.06 & -077.84 & 015.0 & 7.1 & 007 & 64 & 086 & 102 & 012 & $\mathrm{TF}$ \\
\hline 19870617-013301 & -05.53 & 131.20 & 075.4 & 7.1 & 062 & 62 & 047 & 004 & 094 & UF \\
\hline $19870808-154910$ & -18.96 & -070.02 & 079.5 & 7.2 & 353 & 70 & -091 & 175 & 085 & NF \\
\hline $19870903-064036$ & -59.28 & 158.80 & 015.0 & 7.4 & 062 & 83 & -021 & 019 & 109 & SS \\
\hline 19871006-041915 & -18.29 & -171.93 & 015.0 & 7.2 & 203 & 52 & -070 & 008 & 098 & NF \\
\hline $19871012-135717$ & -07.12 & 154.05 & 018.2 & 7.0 & 114 & 51 & -109 & 129 & 039 & NF \\
\hline 19871016-204818 & -06.22 & 149.44 & 047.8 & 7.3 & 086 & 58 & 090 & 176 & 086 & TF \\
\hline $19871117-084705$ & 58.87 & -143.62 & 015.0 & 7.1 & 356 & 85 & -147 & 040 & 130 & SS \\
\hline $19871130-192340$ & 58.17 & -142.04 & 015.0 & 7.8 & 262 & 82 & -020 & 038 & 128 & SS \\
\hline 19880119-073046 & -24.86 & -070.55 & 031.0 & 7.0 & 177 & 73 & 091 & 085 & 175 & $\mathrm{TF}$ \\
\hline $19880205-140117$ & -24.93 & -070.56 & 017.2 & 7.1 & 181 & 73 & 092 & 087 & 177 & TF \\
\hline 19880204-035214 & 13.42 & 125.03 & 034.7 & 7.2 & 343 & 70 & 094 & 067 & 157 & $\mathrm{TF}$ \\
\hline $19880306-223552$ & 57.37 & -143.53 & 015.0 & 7.7 & 089 & 79 & -015 & 046 & 136 & SS \\
\hline $19880412-232007$ & -17.55 & -072.83 & 015.0 & 7.1 & 145 & 79 & 091 & 052 & 142 & $\mathrm{TF}$ \\
\hline 19880806-003638 & 25.19 & 094.89 & 100.5 & 7.2 & 148 & 54 & 120 & 036 & 126 & $\mathrm{TF}$ \\
\hline $19880810-043844$ & -10.49 & 160.77 & 016.2 & 7.5 & 138 & 73 & 081 & 062 & 152 & $\mathrm{TF}$ \\
\hline $19881008-044636$ & -18.82 & -172.28 & 045.0 & 7.1 & 16 & 47 & 090 & 106 & 016 & $\mathrm{TF}$ \\
\hline 19881106-130331 & 23.00 & 099.68 & 015.0 & 7.0 & 064 & 84 & 012 & 018 & 108 & SS \\
\hline $19890210-111531$ & 02.64 & 126.72 & 043.5 & 7.1 & 210 & 59 & 092 & 118 & 028 & $\mathrm{TF}$ \\
\hline $19890505-182849$ & -08.05 & -071.49 & 606.0 & 7.1 & 157 & 60 & -093 & 160 & 070 & $\mathrm{NF}$ \\
\hline
\end{tabular}


Table 1. (Continued.)

\begin{tabular}{|c|c|c|c|c|c|c|c|c|c|c|}
\hline \multirow[b]{2}{*}{ Time } & \multicolumn{3}{|c|}{ Location } & \multicolumn{4}{|c|}{ Focal mechanism data } & \multicolumn{2}{|c|}{$\mathrm{SH}$} & \multirow{2}{*}{$\begin{array}{c}\text { Stress } \\
\text { Regime }\end{array}$} \\
\hline & Lat. & Long. & Depth & $M w$ & Strike & Dip & Slip & Max & Min & \\
\hline $19890523-105512$ & -52.15 & 160.41 & 015.0 & 8.0 & 127 & 81 & 021 & 080 & 170 & SS \\
\hline 19890904-131509 & 55.66 & -157.23 & 025.7 & 7.0 & 062 & 82 & 089 & 156 & 066 & UF \\
\hline $19891101-182542$ & 39.95 & 143.08 & 024.0 & 7.4 & 025 & 77 & 095 & 104 & 014 & $\mathrm{TF}$ \\
\hline $19891215-184408$ & 07.88 & 126.96 & 036.9 & 7.5 & 025 & 59 & 106 & 100 & 010 & $\mathrm{TF}$ \\
\hline $19900303-121644$ & -22.05 & 175.35 & 025.3 & 7.6 & 137 & 87 & 158 & 003 & 093 & SS \\
\hline $19900305-163822$ & -18.35 & 168.04 & 036.9 & 7.0 & 153 & 63 & 083 & 070 & 160 & $\mathrm{TF}$ \\
\hline $19900325-132308$ & 09.95 & -084.58 & 017.9 & 7.3 & 108 & 79 & 087 & 025 & 115 & $\mathrm{TF}$ \\
\hline $19900405-211247$ & 15.57 & 148.08 & 015.0 & 7.4 & 026 & 61 & -080 & 016 & 106 & NF \\
\hline $19900418-133936$ & 01.31 & 123.35 & 033.2 & 7.6 & 257 & 64 & 073 & 005 & 95 & $\mathrm{TF}$ \\
\hline $19900512-045017$ & 48.94 & 041.38 & 612.5 & 7.2 & 056 & 76 & -064 & 024 & 114 & UF \\
\hline $19900520-022208$ & 05.32 & 032.29 & 015.0 & 7.1 & 315 & 86 & 023 & 089 & 179 & SS \\
\hline 19900524-200022 & 05.70 & 031.67 & 015.0 & 7.1 & 102 & 59 & -059 & 077 & 167 & NF \\
\hline 19900524-200931 & -07.30 & 120.95 & 580.3 & 7.0 & 245 & 66 & -082 & 056 & 146 & NF \\
\hline 19900614-074108 & 11.70 & 121.86 & 015.0 & 7.0 & 132 & 79 & -013 & 088 & 178 & SS \\
\hline 19900620-210031 & 36.95 & 049.52 & 015.0 & 7.4 & 300 & 73 & 032 & 070 & 160 & SS \\
\hline $19900716-072653$ & 15.97 & 121.23 & 015.0 & 7.7 & 333 & 88 & 004 & 108 & 018 & SS \\
\hline $19900727-123807$ & -15.50 & 167.26 & 129.0 & 7.2 & 194 & 59 & 115 & 082 & 172 & $\mathrm{TF}$ \\
\hline $19900812-212535$ & -19.58 & 169.05 & 164.9 & 7.0 & 165 & 74 & 095 & 066 & 156 & $\mathrm{TF}$ \\
\hline $19901106-201440$ & 53.77 & 169.41 & 026.6 & 7.1 & 057 & 82 & 069 & 002 & 092 & UF \\
\hline $19901230-191436$ & -05.23 & 150.73 & 204.8 & 7.4 & 102 & 80 & 127 & 153 & 063 & UF \\
\hline $19910422-215712$ & 10.10 & -082.77 & 015.0 & 7.6 & 318 & 69 & 104 & 030 & 120 & $\mathrm{TF}$ \\
\hline $19910620-051901$ & 01.04 & 123.23 & 015.0 & 7.5 & 276 & 83 & 088 & 014 & 104 & UF \\
\hline $19910623-212243$ & -27.23 & -063.25 & 570.3 & 7.2 & 162 & 68 & -104 & 179 & 089 & NF \\
\hline $19910817-221721$ & 41.71 & -125.63 & 015.0 & 7.0 & 046 & 86 & 028 & 00 & 090 & SS \\
\hline $19911014-155825$ & -09.17 & 158.36 & 030.3 & 7.2 & 163 & 68 & 109 & 052 & 142 & $\mathrm{TF}$ \\
\hline $19911119-222901$ & 04.80 & -077.18 & 019.1 & 7.2 & 188 & 77 & 089 & 100 & 010 & $\mathrm{TF}$ \\
\hline $19911222-084331$ & 45.58 & 151.55 & 031.2 & 7.6 & 037 & 74 & 088 & 131 & 041 & $\mathrm{TF}$ \\
\hline $19911227-040612$ & -55.91 & -024.48 & 015.0 & 7.1 & 141 & 59 & -090 & 141 & 051 & NF \\
\hline $19920425-180612$ & 40.25 & -124.31 & 015.0 & 7.2 & 173 & 82 & 093 & 071 & 161 & UF \\
\hline $19920515-070518$ & -06.35 & 147.59 & 039.8 & 7.2 & 104 & 56 & 091 & 013 & 103 & $\mathrm{TF}$ \\
\hline 19920517-094930 & 07.27 & 126.96 & 034.0 & 7.1 & 034 & 67 & 109 & 103 & 013 & $\mathrm{TF}$ \\
\hline $19920517-101542$ & 07.33 & 127.18 & 024.5 & 7.2 & 020 & 67 & 100 & 099 & 009 & $\mathrm{TF}$ \\
\hline $19920527-051346$ & -11.20 & 165.06 & 018.7 & 7.0 & 340 & 56 & -094 & 164 & 074 & NF \\
\hline $19920628-115753$ & 34.65 & -116.65 & 015.0 & 7.3 & 248 & 82 & -020 & 024 & 114 & SS \\
\hline $19920711-104433$ & -22.45 & -177.96 & 393.8 & 7.2 & 029 & 77 & -078 & 007 & 097 & NF \\
\hline $19920819-020446$ & 42.19 & 073.32 & 017.0 & 7.2 & 088 & 60 & 099 & 169 & 079 & $\mathrm{TF}$ \\
\hline $19920902-001642$ & 11.20 & -087.81 & 015.0 & 7.6 & 122 & 78 & 090 & 032 & 122 & $\mathrm{TF}$ \\
\hline $19921011-192438$ & -19.17 & 168.83 & 141.1 & 7.4 & 185 & 78 & 123 & 059 & 149 & UF \\
\hline $19921018-151210$ & 07.27 & -076.34 & 015.0 & 7.1 & 009 & 81 & 046 & 139 & 049 & UF \\
\hline $19921212-052950$ & -08.34 & 122.49 & 020.4 & 7.7 & 253 & 50 & 086 & 166 & 076 & $\mathrm{TF}$ \\
\hline $19921220-205301$ & -06.60 & 130.52 & 069.7 & 7.2 & 335 & 61 & 137 & 032 & 122 & $\mathrm{TS}$ \\
\hline $19930115-110616$ & 43.06 & 144.29 & 100.0 & 7.6 & 259 & 86 & -095 & 102 & 012 & UF \\
\hline 19930306-030600 & -10.73 & 164.02 & 015.0 & 7.1 & 158 & 84 & 145 & 025 & 115 & SS \\
\hline $19930511-182700$ & 07.07 & 127.00 & 046.5 & 7.0 & 031 & 51 & 114 & 103 & 013 & $\mathrm{TF}$ \\
\hline $19930608-130357$ & 51.36 & 158.75 & 045.9 & 7.5 & 039 & 61 & 096 & 123 & 033 & $\mathrm{TF}$ \\
\hline $19930712-131737$ & 42.71 & 139.28 & 016.5 & 7.7 & 179 & 55 & 090 & 089 & 179 & $\mathrm{TF}$ \\
\hline $19930808-083449$ & 13.06 & 145.31 & 059.3 & 7.7 & 075 & 80 & 075 & 014 & 104 & $\mathrm{TF}$ \\
\hline $19930809-124258$ & 36.48 & 070.47 & 213.7 & 7.0 & 109 & 67 & 098 & 009 & 099 & $\mathrm{TF}$ \\
\hline $19930910-191303$ & 14.41 & -092.99 & 029.1 & 7.2 & 124 & 67 & 096 & 026 & 116 & $\mathrm{TF}$ \\
\hline 19931113-011816 & 52.00 & 159.27 & 054.2 & 7.0 & 034 & 59 & 094 & 120 & 030 & $\mathrm{TF}$ \\
\hline $19931229-074824$ & -20.39 & 169.94 & 015.0 & 7.0 & 302 & 73 & -146 & 162 & 072 & SS \\
\hline $19940309-232818$ & -17.69 & -178.11 & 567.8 & 7.6 & 007 & 77 & -114 & 039 & 129 & UF \\
\hline
\end{tabular}


Table 1. (Continued.)

\begin{tabular}{|c|c|c|c|c|c|c|c|c|c|c|}
\hline \multirow[b]{2}{*}{ Time } & \multicolumn{3}{|c|}{ Location } & \multicolumn{4}{|c|}{ Focal mechanism data } & \multicolumn{2}{|c|}{$\mathrm{SH}$} & \multirow{2}{*}{$\begin{array}{c}\text { Stress } \\
\text { Regime }\end{array}$} \\
\hline & Lat. & Long. & Depth & $M w$ & Strike & Dip & Slip & Max & Min & \\
\hline 19940314-043033 & -00.88 & -023.03 & 015.0 & 7.0 & 171 & 88 & -029 & 127 & 037 & SS \\
\hline $19940602-181816$ & -11.03 & 113.04 & 015.0 & 7.8 & 099 & 83 & 090 & 009 & 099 & UF \\
\hline 19940609-003345 & -13.82 & -067.25 & 647.1 & 8.2 & 092 & 81 & -095 & 106 & 016 & UF \\
\hline 19940713-023609 & -16.50 & 167.35 & 025.0 & 7.1 & 181 & 89 & 132 & 047 & 137 & UF \\
\hline $19940721-183640$ & 42.30 & 133.04 & 489.1 & 7.3 & 155 & 89 & 056 & 110 & 020 & UF \\
\hline 19940901-151601 & 40.59 & -125.78 & 015.0 & 7.0 & 06 & 86 & 025 & 140 & 050 & SS \\
\hline 19941004-132329 & 43.60 & 147.63 & 068.2 & 8.3 & 050 & 74 & 128 & 104 & 014 & UF \\
\hline 19941009-075549 & 43.87 & 147.96 & 033.2 & 7.2 & 031 & 72 & 085 & 129 & 039 & $\mathrm{TF}$ \\
\hline 19941114-191539 & 13.44 & 121.32 & 015.0 & 7.1 & 249 & 88 & -020 & 025 & 115 & SS \\
\hline $19941228-121959$ & 40.56 & 142.99 & 027.7 & 7.7 & 022 & 79 & 95 & 100 & 010 & $\mathrm{TF}$ \\
\hline $19950205-225113$ & -37.61 & 179.40 & 015.0 & 7.1 & 215 & 58 & -090 & 035 & 125 & NF \\
\hline $19950407-220714$ & -15.37 & -173.15 & 087.9 & 7.3 & 298 & 84 & 073 & 064 & 154 & UF \\
\hline $19950421-003500$ & 12.27 & 125.69 & 021.7 & 7.1 & 003 & 71 & 101 & 077 & 167 & $\mathrm{TF}$ \\
\hline $19950505-035359$ & 12.67 & 125.30 & 018.3 & 7.0 & 351 & 76 & 097 & 67 & 157 & $\mathrm{TF}$ \\
\hline 19950516-201302 & -23.05 & 170.00 & 024.7 & 7.7 & 110 & 56 & -084 & 105 & 015 & NF \\
\hline $19950527-130403$ & 53.03 & 142.65 & 023.6 & 7.0 & 196 & 82 & 169 & 062 & 152 & SS \\
\hline $19950703-195103$ & -29.13 & -177.22 & 044.7 & 7.1 & 011 & 62 & 087 & 104 & 014 & $\mathrm{TF}$ \\
\hline $19950730-051157$ & -24.17 & -070.74 & 028.7 & 8.0 & 177 & 68 & 091 & 085 & 175 & $\mathrm{TF}$ \\
\hline $19950816-102801$ & -05.51 & 153.64 & 045.6 & 7.7 & 320 & 48 & 092 & 049 & 139 & $\mathrm{TF}$ \\
\hline $19950816-231048$ & -06.08 & 154.19 & 045.0 & 7.2 & 311 & 45 & 089 & 042 & 132 & $\mathrm{TF}$ \\
\hline 19950823-070609 & 18.88 & 145.30 & 599.2 & 7.0 & 351 & 53 & -067 & 153 & 063 & NF \\
\hline $19950914-140441$ & 16.73 & -098.54 & 021.8 & 7.3 & 115 & 75 & 091 & 023 & 113 & $\mathrm{TF}$ \\
\hline $19951003-015137$ & -02.55 & -077.53 & 025.0 & 7.0 & 018 & 57 & 068 & 127 & 037 & $\mathrm{TF}$ \\
\hline 19951009-153629 & 19.34 & -104.80 & 015.0 & 8.0 & 120 & 81 & 090 & 030 & 120 & UF \\
\hline 19951018-103739 & 28.06 & 130.18 & 018.5 & 7.1 & 212 & 66 & -076 & 016 & 106 & NF \\
\hline 19951021-023909 & 16.67 & -093.42 & 163.8 & 7.2 & 313 & 90 & -104 & 178 & 088 & UF \\
\hline $19951122-041526$ & 29.07 & 034.73 & 018.4 & 7.2 & 294 & 77 & -149 & 155 & 065 & SS \\
\hline $19951203-180136$ & 44.82 & 150.17 & 025.9 & 7.9 & 040 & 78 & 089 & 132 & 042 & $\mathrm{TF}$ \\
\hline $19951225-044336$ & -06.93 & 129.65 & 160.7 & 7.0 & 033 & 64 & 049 & 155 & 065 & UF \\
\hline 19960101-080523 & 00.74 & 119.93 & 015.0 & 7.9 & 252 & 85 & 094 & 145 & 055 & UF \\
\hline $19960207-213657$ & 45.29 & 150.45 & 048.7 & 7.1 & 030 & 64 & 078 & 133 & 043 & $\mathrm{TF}$ \\
\hline $19960217-060003$ & -00.67 & 136.62 & 015.0 & 8.2 & 305 & 80 & 094 & 023 & 113 & UF \\
\hline $19960221-125127$ & -09.95 & -080.23 & 015.0 & 7.5 & 157 & 76 & 091 & 065 & 155 & $\mathrm{TF}$ \\
\hline 19960225-030827 & 15.88 & -097.98 & 015.0 & 7.1 & 117 & 74 & 095 & 018 & 108 & $\mathrm{TF}$ \\
\hline 19960416-003105 & -23.98 & -176.47 & 116.2 & 7.1 & 311 & 75 & 123 & 007 & 097 & UF \\
\hline 19960429-144051 & -06.65 & 155.07 & 054.3 & 7.2 & 305 & 46 & 081 & 041 & 131 & $\mathrm{TF}$ \\
\hline 19960610-040403 & 51.10 & -177.41 & 029.0 & 7.9 & 074 & 74 & 092 & 160 & 070 & $\mathrm{TF}$ \\
\hline 19960610-152509 & 51.38 & -176.49 & 036.5 & 7.2 & 062 & 66 & 083 & 160 & 070 & $\mathrm{TF}$ \\
\hline $19960611-182306$ & 12.74 & 125.41 & 028.5 & 7.1 & 356 & 70 & 102 & 070 & 160 & TF \\
\hline $19960617-112234$ & -07.38 & 123.02 & 584.2 & 7.8 & 098 & 54 & -052 & 071 & 161 & NF \\
\hline $19960722-141948$ & 01.34 & 120.65 & 028.2 & 7.0 & 264 & 77 & 096 & 161 & 071 & $\mathrm{TF}$ \\
\hline $19960805-223831$ & -20.72 & -178.16 & 555.0 & 7.4 & 043 & 70 & -087 & 039 & 129 & NF \\
\hline $19961112-170016$ & -15.04 & -075.37 & 037.4 & 7.7 & 172 & 64 & 110 & 062 & 152 & $\mathrm{TF}$ \\
\hline $19970100-202840$ & 18.34 & -102.58 & 040.0 & 7.1 & 292 & 82 & -106 & 145 & 055 & UF \\
\hline $19970123-021532$ & -22.04 & -065.92 & 281.6 & 7.1 & 351 & 90 & -086 & 127 & 037 & UF \\
\hline $19970227-210814$ & 29.74 & 068.13 & 015.3 & 7.1 & 085 & 77 & 082 & 012 & 102 & $\mathrm{TF}$ \\
\hline 19970421-120309 & -13.21 & 166.20 & 051.2 & 7.7 & 178 & 66 & 122 & 059 & 149 & $\mathrm{TF}$ \\
\hline $19970510-075750$ & 33.58 & 060.02 & 015.0 & 7.2 & 338 & 90 & -173 & 023 & 113 & SS \\
\hline $19970525-232244$ & -32.02 & -179.95 & 345.0 & 7.1 & 342 & 75 & 049 & 109 & 019 & UF \\
\hline $19970920-161143$ & -28.83 & -176.99 & 046.4 & 7.0 & 015 & 61 & 090 & 105 & 015 & $\mathrm{TF}$ \\
\hline 19971014-095333 & -21.94 & -176.15 & 165.9 & 7.7 & 016 & 81 & -105 & 046 & 136 & UF \\
\hline
\end{tabular}


Table 1. (Continued.)

\begin{tabular}{|c|c|c|c|c|c|c|c|c|c|c|}
\hline \multirow[b]{2}{*}{ Time } & \multicolumn{3}{|c|}{ Location } & \multicolumn{4}{|c|}{ Focal mechanism data } & \multicolumn{2}{|c|}{$\mathrm{SH}$} & \multirow{2}{*}{$\begin{array}{c}\text { Stress } \\
\text { Regime }\end{array}$} \\
\hline & Lat. & Long. & Depth & $M w$ & Strike & Dip & Slip & Max & Min & \\
\hline $19971015-010343$ & -31.06 & -071.42 & 069.8 & 7.1 & 173 & 80 & -083 & 156 & 066 & UF \\
\hline $19971028-061528$ & -04.44 & -076.55 & 118.9 & 7.2 & 158 & 52 & -091 & 159 & 069 & NF \\
\hline 19971108-100303 & 35.33 & 086.96 & 016.4 & 7.5 & 348 & 88 & 159 & 033 & 123 & SS \\
\hline $19971115-185933$ & -14.92 & 167.21 & 121.8 & 7.0 & 197 & 50 & 103 & 097 & 007 & $\mathrm{TF}$ \\
\hline $19971125-121443$ & 01.37 & 122.71 & 029.4 & 7.0 & 275 & 69 & 089 & 007 & 097 & $\mathrm{TF}$ \\
\hline $19971205-112721$ & 54.31 & 161.91 & 033.6 & 7.8 & 039 & 68 & 097 & 120 & 030 & $\mathrm{TF}$ \\
\hline 19971222-020602 & -05.56 & 148.05 & 196.5 & 7.1 & 221 & 75 & -024 & 180 & 090 & SS \\
\hline $19980104-061226$ & -22.31 & 171.08 & 114.3 & 7.4 & 092 & 76 & 045 & 040 & 130 & $\mathrm{TS}$ \\
\hline $19980130-121620$ & -24.02 & -070.62 & 041.0 & 7.0 & 172 & 74 & 086 & 088 & 178 & $\mathrm{TF}$ \\
\hline 19980325-031303 & -62.99 & 148.64 & 028.8 & 8.1 & 281 & 84 & 017 & 055 & 145 & SS \\
\hline $19980329-194823$ & -17.57 & -178.85 & 553.7 & 7.1 & 104 & 71 & 040 & 052 & 142 & $\mathrm{TS}$ \\
\hline $19980503-233038$ & 22.37 & 125.53 & 022.9 & 7.4 & 049 & 89 & 172 & 094 & 004 & SS \\
\hline $19980716-115642$ & -10.91 & 166.09 & 100.3 & 7.0 & 198 & 54 & 078 & 118 & 028 & $\mathrm{TF}$ \\
\hline $19980717-084933$ & -02.50 & 142.07 & 015.0 & 7.0 & 287 & 75 & 078 & 037 & 127 & $\mathrm{TF}$ \\
\hline 19980804-185929 & -00.57 & -080.48 & 025.6 & 7.1 & 172 & 78 & 082 & 100 & 010 & $\mathrm{TF}$ \\
\hline $19980820-064111$ & 28.99 & 139.47 & 425.5 & 7.0 & 337 & 82 & -064 & 120 & 030 & UF \\
\hline 19981109-053849 & -06.94 & 128.95 & 024.6 & 7.0 & 084 & 56 & 075 & 007 & 097 & $\mathrm{TF}$ \\
\hline $19981129-141045$ & -02.03 & 125.00 & 016.4 & 7.7 & 196 & 65 & -150 & 054 & 144 & SS \\
\hline 19990119-033543 & -04.72 & 153.66 & 087.5 & 7.0 & 326 & 59 & 092 & 054 & 144 & $\mathrm{TF}$ \\
\hline 19990206-214809 & -12.78 & 166.58 & 098.2 & 7.3 & 178 & 69 & 116 & 061 & 151 & $\mathrm{TF}$ \\
\hline 19990304-085211 & 05.38 & 122.03 & 018.0 & 7.1 & 167 & 54 & 094 & 073 & 163 & $\mathrm{TF}$ \\
\hline $19990405-110817$ & -05.65 & 149.71 & 149.4 & 7.4 & 094 & 75 & 097 & 171 & 081 & $\mathrm{TF}$ \\
\hline $19990408-131040$ & 43.66 & 130.47 & 575.4 & 7.1 & 189 & 82 & 067 & 135 & 045 & UF \\
\hline $19990510-203310$ & -05.38 & 150.97 & 144.6 & 7.0 & 069 & 54 & -058 & 046 & 136 & NF \\
\hline 19990516-005132 & -04.99 & 152.76 & 042.0 & 7.0 & 075 & 62 & 087 & 168 & 078 & $\mathrm{TF}$ \\
\hline 19990817-000150 & 41.01 & 029.97 & 017.0 & 7.6 & 091 & 87 & 164 & 137 & 047 & SS \\
\hline $19990920-174735$ & 24.15 & 120.80 & 021.2 & 7.6 & 211 & 65 & 087 & 124 & 034 & $\mathrm{TF}$ \\
\hline 19990930-163123 & 16.20 & -096.96 & 046.8 & 7.4 & 300 & 49 & -078 & 112 & 022 & NF \\
\hline 19991016-094659 & 34.71 & -116.27 & 015.0 & 7.1 & 067 & 85 & 010 & 021 & 111 & SS \\
\hline $19991112-165728$ & 40.93 & 031.25 & 018.0 & 7.1 & 170 & 80 & -036 & 129 & 039 & SS \\
\hline 19991119-135702 & -06.49 & 148.98 & 033.0 & 7.0 & 084 & 66 & 085 & 00 & 090 & $\mathrm{TF}$ \\
\hline $19991126-132145$ & -16.08 & 168.31 & 015.0 & 7.4 & 020 & 62 & 103 & 096 & 006 & $\mathrm{TF}$ \\
\hline $19991206-231240$ & 57.35 & -154.35 & 054.1 & 7.0 & 267 & 90 & -027 & 042 & 132 & SS \\
\hline $19991211-180346$ & 15.87 & 119.64 & 035.1 & 7.2 & 011 & 88 & -077 & 152 & 062 & UF \\
\hline $20000108-164730$ & -16.84 & -173.81 & 162.4 & 7.2 & 182 & 88 & -098 & 041 & 131 & UF \\
\hline 20000225-014405 & -19.55 & 174.17 & 016.8 & 7.1 & 047 & 80 & 016 & 001 & 091 & SS \\
\hline 20000328-110031 & 22.32 & 143.76 & 099.7 & 7.6 & 112 & 85 & -086 & 095 & 005 & $\mathrm{UF}$ \\
\hline 20000504-042133 & -01.29 & 123.59 & 018.6 & 7.5 & 319 & 83 & 026 & 093 & 003 & SS \\
\hline $20000512-184329$ & -23.72 & -066.85 & 226.6 & 7.1 & 005 & 80 & -096 & 020 & 110 & UF \\
\hline 20000604-162847 & -04.73 & 101.94 & 043.9 & 7.8 & 199 & 67 & 038 & 145 & 055 & $\mathrm{TS}$ \\
\hline $20000618-144428$ & -13.47 & 097.17 & 015.0 & 7.9 & 254 & 85 & -153 & 118 & 028 & SS \\
\hline 20000806-072724 & 28.89 & 139.68 & 411.3 & 7.3 & 344 & 74 & -068 & 137 & 047 & $\mathrm{NF}$ \\
\hline 20001116-045537 & -04.56 & 152.79 & 024.0 & 8.0 & 236 & 88 & 133 & 102 & 012 & $\mathrm{UF}$ \\
\hline 20001116-074245 & -05.03 & 153.17 & 031.2 & 7.8 & 070 & 75 & 089 & 162 & 072 & $\mathrm{TF}$ \\
\hline $20001117-210220$ & -05.26 & 152.34 & 017.0 & 7.8 & 078 & 68 & 101 & 155 & 065 & $\mathrm{TF}$ \\
\hline 20001206-171115 & 39.60 & 054.87 & 033.0 & 7.0 & 089 & 68 & 065 & 024 & 114 & $\mathrm{TF}$ \\
\hline 20010101-065724 & 06.73 & 127.07 & 044.0 & 7.4 & 030 & 52 & 117 & 100 & 010 & $\mathrm{TF}$ \\
\hline 20010109-164938 & -14.90 & 167.11 & 114.4 & 7.0 & 288 & 76 & 047 & 056 & 146 & $\mathrm{TS}$ \\
\hline 20010113-173346 & 12.97 & -089.13 & 056.0 & 7.7 & 307 & 56 & -086 & 123 & 033 & $\mathrm{NF}$ \\
\hline 20010126-031655 & 23.63 & 070.24 & 019.8 & 7.6 & 066 & 64 & 060 & 002 & 092 & $\mathrm{TF}$ \\
\hline 20010213-192845 & -05.40 & 102.36 & 021.2 & 7.3 & 121 & 74 & 086 & 038 & 128 & $\mathrm{TF}$ \\
\hline 20010224-072358 & 01.55 & 126.42 & 042.8 & 7.0 & 032 & 49 & 100 & 114 & 024 & $\mathrm{TF}$ \\
\hline
\end{tabular}


Table 1. (Continued.)

\begin{tabular}{|c|c|c|c|c|c|c|c|c|c|c|}
\hline \multirow[b]{2}{*}{ Time } & \multicolumn{3}{|c|}{ Location } & \multicolumn{4}{|c|}{ Focal mechanism data } & \multicolumn{2}{|c|}{$\mathrm{SH}$} & \multirow{2}{*}{$\begin{array}{c}\text { Stress } \\
\text { Regime }\end{array}$} \\
\hline & Lat. & Long. & Depth & $M w$ & Strike & Dip & Slip & Max & Min & \\
\hline 20010603-024210 & -29.37 & -178.23 & 199.3 & 7.1 & 351 & 64 & 057 & 109 & 019 & $\mathrm{TF}$ \\
\hline $20010623-203423$ & -17.28 & -072.71 & 029.6 & 8.4 & 159 & 74 & 098 & 056 & 146 & $\mathrm{TF}$ \\
\hline 20010707-093902 & -17.45 & -072.45 & 025.0 & 7.6 & 165 & 79 & 099 & 055 & 145 & TF \\
\hline 20010821-065214 & -36.70 & -179.08 & 059.0 & 7.1 & 202 & 53 & 068 & 129 & 039 & $\mathrm{TF}$ \\
\hline $20011012-150223$ & 12.88 & 145.08 & 042.0 & 7.0 & 013 & 75 & 116 & 072 & 162 & $\mathrm{TF}$ \\
\hline 20011019-032858 & -04.31 & 124.11 & 018.8 & 7.4 & 089 & 86 & 013 & 044 & 134 & SS \\
\hline 20011114-092716 & 35.80 & 092.91 & 015.0 & 7.8 & 190 & 80 & -150 & 052 & 142 & SS \\
\hline $20011212-140250$ & -42.69 & 124.67 & 016.0 & 7.1 & 259 & 85 & 172 & 125 & 035 & SS \\
\hline 20020102-172306 & -17.78 & 167.85 & 040.0 & 7.2 & 189 & 84 & 106 & 064 & 154 & $\mathrm{UF}$ \\
\hline 20020303-120824 & 36.57 & 070.42 & 228.5 & 7.3 & 108 & 68 & 092 & 016 & 106 & $\mathrm{TF}$ \\
\hline 20020305-211623 & 05.92 & 124.25 & 028.7 & 7.5 & 156 & 67 & 099 & 055 & 145 & TF \\
\hline $20020331-065300$ & 24.19 & 121.96 & 039.0 & 7.1 & 077 & 63 & 072 & 005 & 095 & $\mathrm{TF}$ \\
\hline 20020426-160614 & 13.15 & 144.67 & 069.1 & 7.0 & 071 & 89 & 093 & 124 & 034 & UF \\
\hline 20020628-171940 & 43.74 & 130.45 & 581.5 & 7.3 & 192 & 77 & 086 & 110 & 020 & $\mathrm{TF}$ \\
\hline 20020819-110115 & -21.74 & -179.08 & 630.9 & 7.6 & 022 & 76 & -072 & 175 & 085 & NF \\
\hline 20020819-110835 & -24.16 & 178.49 & 699.3 & 7.7 & 237 & 87 & 090 & 147 & 057 & UF \\
\hline 20020908-184438 & -03.27 & 143.38 & 019.5 & 7.6 & 339 & 68 & 116 & 043 & 133 & $\mathrm{TF}$ \\
\hline 20021010-105042 & -01.79 & 134.30 & 015.0 & 7.5 & 329 & 86 & 173 & 014 & 104 & SS \\
\hline 20021102-012626 & 02.65 & 095.99 & 023.0 & 7.2 & 135 & 75 & 095 & 036 & 126 & $\mathrm{TF}$ \\
\hline 20021103-221328 & 63.23 & -144.89 & 015.0 & 7.8 & 029 & 82 & 019 & 162 & 072 & SS \\
\hline $20021117-045400$ & 47.81 & 146.45 & 479.8 & 7.3 & 197 & 86 & 098 & 075 & 165 & UF \\
\hline $20030120-084316$ & -10.43 & 160.63 & 031.0 & 7.2 & 293 & 58 & 041 & 055 & 145 & $\mathrm{TS}$ \\
\hline $20030122-020649$ & 18.86 & -103.90 & 026.0 & 7.5 & 108 & 79 & 086 & 027 & 117 & $\mathrm{TF}$ \\
\hline $20030317-163627$ & 51.33 & 177.58 & 027.0 & 7.0 & 060 & 75 & 073 & 175 & 085 & $\mathrm{TF}$ \\
\hline 20030526-092439 & 38.94 & 141.57 & 061.0 & 7.0 & 193 & 72 & 097 & 091 & 001 & $\mathrm{TF}$ \\
\hline 20030620-061947 & -07.37 & -071.89 & 556.2 & 7.0 & 139 & 49 & -114 & 156 & 066 & NF \\
\hline $20030715-202838$ & -01.42 & 069.47 & 015.0 & 7.5 & 216 & 89 & 159 & 081 & 171 & SS \\
\hline 20030804-043743 & -60.80 & -043.21 & 015.0 & 7.6 & 210 & 76 & -124 & 065 & 155 & UF \\
\hline $20030821-121300$ & -45.01 & 166.87 & 031.8 & 7.2 & 209 & 67 & 088 & 121 & 031 & $\mathrm{TF}$ \\
\hline 20030925-195038 & 42.21 & 143.84 & 028.2 & 8.3 & 028 & 82 & 083 & 139 & 049 & UF \\
\hline $20030925-210820$ & 41.75 & 143.62 & 047.3 & 7.3 & 033 & 72 & 091 & 121 & 031 & $\mathrm{TF}$ \\
\hline 20030927-113336 & 50.02 & 087.86 & 015.0 & 7.2 & 131 & 71 & 158 & 180 & 090 & SS \\
\hline 20031031-010641 & 37.89 & 142.68 & 015.0 & 7.0 & 026 & 81 & 092 & 110 & 020 & UF \\
\hline 20031117-064331 & 51.14 & 177.86 & 021.7 & 7.7 & 067 & 74 & 080 & 173 & 083 & TF \\
\hline $20031227-160122$ & -21.99 & 169.81 & 023.0 & 7.3 & 139 & 61 & 087 & 052 & 142 & $\mathrm{TF}$ \\
\hline 20040103-162331 & -22.41 & 169.72 & 015.0 & 7.1 & 117 & 52 & -100 & 125 & 035 & NF \\
\hline 20040205-210513 & -03.62 & 135.53 & 013.0 & 7.0 & 044 & 43 & -010 & 002 & 092 & UF \\
\hline 20040207-024244 & -04.03 & 134.78 & 012.0 & 7.3 & 261 & 68 & -007 & 038 & 128 & SS \\
\hline $20040715-042720$ & -17.68 & -178.52 & 577.2 & 7.1 & 345 & 44 & 137 & 047 & 137 & $\mathrm{TF}$ \\
\hline $20040725-143525$ & -02.68 & 104.38 & 600.5 & 7.3 & 108 & 45 & -129 & 133 & 043 & NF \\
\hline 20040905-100716 & 32.94 & 137.00 & 016.0 & 7.2 & 277 & 38 & 100 & 001 & 091 & $\mathrm{TF}$ \\
\hline 20040905-145743 & 33.13 & 137.22 & 012.0 & 7.4 & 079 & 46 & 072 & 002 & 092 & TF \\
\hline $20041111-212658$ & -07.87 & 125.12 & 017.0 & 7.5 & 067 & 27 & 072 & 167 & 077 & $\mathrm{TF}$ \\
\hline 20041115-090702 & 04.72 & -077.57 & 016.0 & 7.2 & 021 & 11 & 114 & 099 & 009 & UF \\
\hline $20041122-202635$ & -46.36 & 164.91 & 040.0 & 7.1 & 043 & 36 & 103 & 125 & 035 & $\mathrm{TF}$ \\
\hline $20041126-022512$ & -03.55 & 135.54 & 012.0 & 7.1 & 005 & 34 & 00 & 140 & 050 & UF \\
\hline 20041128-183219 & 42.88 & 145.36 & 047.0 & 7.0 & 242 & 26 & 122 & 134 & 044 & $\mathrm{TF}$ \\
\hline $20041223-145931$ & -49.91 & 161.25 & 027.5 & 8.1 & 069 & 74 & 167 & 116 & 026 & SS \\
\hline 20041226-010109 & 03.09 & 094.26 & 028.6 & 9.0 & 329 & 08 & 110 & 047 & 137 & UF \\
\hline $20041226-042137$ & 06.61 & 092.79 & 013.6 & 7.2 & 351 & 27 & 121 & 064 & 154 & $\mathrm{TF}$ \\
\hline $20050205-122324$ & 05.47 & 123.67 & 530.6 & 7.1 & 158 & 14 & -114 & 171 & 081 & NF \\
\hline $20050302-104217$ & -06.54 & 129.99 & 196.1 & 7.1 & 041 & 88 & 055 & 175 & 085 & UF \\
\hline
\end{tabular}


Table 1. (Continued.)

\begin{tabular}{|c|c|c|c|c|c|c|c|c|c|c|}
\hline \multirow[b]{2}{*}{ Time } & \multicolumn{3}{|c|}{ Location } & \multicolumn{4}{|c|}{ Focal mechanism data } & \multicolumn{2}{|c|}{$\mathrm{SH}$} & \multirow{2}{*}{$\begin{array}{c}\text { Stress } \\
\text { Regime }\end{array}$} \\
\hline & Lat. & Long. & Depth & $M w$ & Strike & Dip & Slip & Max & Min & \\
\hline 20050328-161032 & 01.67 & 097.07 & 025.8 & 8.6 & 125 & 83 & 086 & 051 & 141 & UF \\
\hline $20050613-224440$ & -20.02 & -069.23 & 094.5 & 7.8 & 352 & 67 & -094 & 177 & 087 & NF \\
\hline 20050615-025105 & 41.15 & -126.42 & 020.4 & 7.2 & 047 & 85 & 007 & 001 & 091 & SS \\
\hline 20050724-154216 & 07.92 & 091.88 & 012.0 & 7.2 & 120 & 89 & -158 & 165 & 075 & SS \\
\hline 20050816-024640 & 38.24 & 142.05 & 037.0 & 7.2 & 024 & 74 & 093 & 109 & 019 & $\mathrm{TF}$ \\
\hline 20050909-072713 & -05.20 & 153.95 & 083.6 & 7.6 & 319 & 60 & 089 & 050 & 140 & $\mathrm{TF}$ \\
\hline 20050926-015544 & -05.60 & -076.20 & 108.1 & 7.5 & 169 & 51 & -088 & 168 & 078 & NF \\
\hline 20051008-035052 & 34.38 & 073.47 & 012.0 & 7.6 & 114 & 57 & 065 & 045 & 135 & $\mathrm{TF}$ \\
\hline 20051114-213859 & 38.22 & 144.97 & 018.0 & 7.0 & 019 & 49 & -078 & 011 & 101 & NF \\
\hline 20060102-061103 & -61.12 & -021.39 & 020.2 & 7.4 & 265 & 78 & -013 & 041 & 131 & SS \\
\hline $20060102-221345$ & -19.80 & -177.72 & 589.5 & 7.2 & 049 & 76 & -107 & 075 & 165 & NF \\
\hline $20060127-165905$ & -05.61 & 128.20 & 397.4 & 7.6 & 169 & 63 & -123 & 016 & 106 & NF \\
\hline $20060222-221915$ & -21.20 & 033.33 & 012.0 & 7.0 & 172 & 65 & -078 & 158 & 068 & NF \\
\hline $20060420-232518$ & 60.89 & 167.05 & 012.0 & 7.6 & 044 & 51 & 101 & 125 & 035 & $\mathrm{TF}$ \\
\hline 20060503-152704 & -20.39 & -173.47 & 067.8 & 8.0 & 011 & 72 & 078 & 119 & 029 & $\mathrm{TF}$ \\
\hline 20060516-103936 & -31.41 & -178.91 & 151.1 & 7.4 & 342 & 74 & 100 & 056 & 146 & $\mathrm{TF}$ \\
\hline $20060717-082038$ & -10.28 & 107.78 & 020.0 & 7.7 & 098 & 80 & 088 & 014 & 104 & UF \\
\hline $20060820-034156$ & -61.27 & -034.52 & 017.2 & 7.0 & 273 & 86 & -008 & 048 & 138 & SS \\
\hline 20061115-111508 & 13.50 & 154.33 & 013.5 & 8.3 & 033 & 75 & 089 & 125 & 035 & $\mathrm{TF}$ \\
\hline 20061226-122629 & 21.81 & 120.52 & 019.6 & 7.0 & 329 & 61 & -098 & 157 & 067 & NF \\
\hline $20070113-042348$ & 46.17 & 154.80 & 012.0 & 8.1 & 266 & 39 & -054 & 064 & 154 & NF \\
\hline $20070121-112801$ & 01.10 & 126.21 & 022.2 & 7.5 & 034 & 35 & 108 & 113 & 023 & $\mathrm{TF}$ \\
\hline 20070325-004013 & -20.60 & 169.12 & 041.2 & 7.1 & 333 & 36 & 089 & 064 & 154 & $\mathrm{TF}$ \\
\hline 20070401-204039 & -07.79 & 156.34 & 014.1 & 8.1 & 333 & 37 & 121 & 044 & 134 & $\mathrm{TF}$ \\
\hline 20070801-170904 & -15.41 & 167.41 & 126.0 & 7.2 & 356 & 29 & 079 & 092 & 002 & $\mathrm{TF}$ \\
\hline 20070808-170511 & -06.03 & 107.58 & 304.8 & 7.5 & 330 & 30 & 155 & 027 & 117 & UF \\
\hline $20070815-234158$ & -13.73 & -077.04 & 033.8 & 8.0 & 321 & 28 & 063 & 066 & 156 & $\mathrm{TF}$ \\
\hline 20070902-010529 & -11.74 & 165.68 & 018.3 & 7.2 & 338 & 23 & 101 & 063 & 153 & $\mathrm{TF}$ \\
\hline $20070912-111116$ & -03.78 & 100.99 & 024.4 & 8.5 & 328 & 09 & 114 & 046 & 136 & UF \\
\hline $20070912-234935$ & -02.46 & 100.13 & 043.1 & 7.9 & 317 & 19 & 102 & 040 & 130 & $\mathrm{TF}$ \\
\hline 20070913-033537 & -02.31 & 099.39 & 017.0 & 7.0 & 312 & 10 & 090 & 042 & 132 & UF \\
\hline 20070928-133904 & 21.94 & 143.07 & 275.8 & 7.5 & 073 & 49 & 050 & 009 & 099 & $\mathrm{TF}$ \\
\hline 20070930-052352 & -49.26 & 164.10 & 012.6 & 7.4 & 029 & 36 & 123 & 100 & 010 & $\mathrm{TF}$ \\
\hline 20071031-033026 & 18.83 & 145.59 & 211.0 & 7.2 & 196 & 60 & 152 & 068 & 158 & UF \\
\hline 20071114-154111 & -22.64 & -070.62 & 037.6 & 7.7 & 358 & 20 & 098 & 083 & 173 & $\mathrm{TF}$ \\
\hline 20071129-190033 & 15.06 & -061.41 & 147.9 & 7.4 & 109 & 58 & 032 & 055 & 145 & $\mathrm{TS}$ \\
\hline 20071209-072838 & -25.75 & -177.22 & 149.9 & 7.8 & 034 & 25 & 038 & 152 & 062 & $\mathrm{TF}$ \\
\hline 20071219-093037 & 51.02 & -179.27 & 027.6 & 7.2 & 274 & 21 & 118 & 169 & 079 & $\mathrm{TF}$ \\
\hline $20080220-080845$ & 02.69 & 095.98 & 014.9 & 7.3 & 299 & 11 & 080 & 034 & 124 & TF \\
\hline $20080225-083642$ & -02.66 & 099.95 & 014.4 & 7.2 & 317 & 06 & 102 & 040 & 130 & UF \\
\hline 20080320-223312 & 35.43 & 081.37 & 012.0 & 7.1 & 358 & 41 & -110 & 011 & 101 & NF \\
\hline 20080409-124624 & -20.12 & 168.80 & 035.2 & 7.3 & 340 & 30 & 101 & 064 & 154 & TF \\
\hline 20080412-003021 & -55.56 & 158.49 & 022.0 & 7.1 & 003 & 43 & 102 & 085 & 175 & $\mathrm{TF}$ \\
\hline $20080512-062840$ & 31.44 & 104.10 & 012.8 & 7.9 & 231 & 35 & 138 & 114 & 024 & $\mathrm{TF}$ \\
\hline 20080630-061752 & -58.33 & -021.77 & 026.0 & 7.0 & 143 & 83 & -179 & 008 & 098 & SS \\
\hline 20080705-021214 & 54.12 & 153.37 & 610.8 & 7.7 & 143 & 48 & -134 & 170 & 080 & NF \\
\hline 20080929-151943 & -29.85 & -177.46 & 051.1 & 7.0 & 197 & 32 & 092 & 106 & 016 & $\mathrm{TF}$ \\
\hline 20081116-170244 & 01.50 & 122.05 & 029.2 & 7.3 & 092 & 20 & 084 & 005 & 095 & $\mathrm{TF}$ \\
\hline 20081124-090310 & 54.27 & 154.71 & 502.3 & 7.3 & 276 & 19 & -029 & 065 & 155 & UF \\
\hline 20090103-194409 & -00.38 & 132.83 & 015.2 & 7.7 & 099 & 23 & 047 & 032 & 122 & $\mathrm{TF}$ \\
\hline 20090103-223345 & -00.58 & 133.48 & 018.2 & 7.4 & 101 & 26 & 072 & 022 & 112 & $\mathrm{TF}$ \\
\hline 20090115-174948 & 46.97 & 155.39 & 045.2 & 7.4 & 011 & 44 & 084 & 105 & 015 & $\mathrm{TF}$ \\
\hline
\end{tabular}


Table 1. (Continued.)

\begin{tabular}{|c|c|c|c|c|c|c|c|c|c|c|}
\hline \multirow[b]{2}{*}{ Time } & \multicolumn{3}{|c|}{ Location } & \multicolumn{4}{|c|}{ Focal mechanism data } & \multicolumn{2}{|c|}{$\mathrm{SH}$} & \multirow{2}{*}{$\begin{array}{c}\text { Stress } \\
\text { Regime }\end{array}$} \\
\hline & Lat. & Long. & Depth & $M w$ & Strike & Dip & Slip & Max & Min & \\
\hline 20090211-173501 & 03.92 & 126.81 & 023.9 & 7.1 & 117 & 38 & 083 & 032 & 122 & $\mathrm{TF}$ \\
\hline $20090218-215350$ & -27.27 & -175.90 & 036.5 & 7.0 & 274 & 38 & 179 & 139 & 049 & UF \\
\hline 20090319-181753 & -23.08 & -174.23 & 049.1 & 7.6 & 205 & 44 & 098 & 109 & 019 & TF \\
\hline 20090528-082505 & 16.50 & -087.17 & 012.0 & 7.3 & 063 & 60 & -007 & 020 & 110 & SS \\
\hline 20090715-092250 & -45.85 & 166.26 & 023.5 & 7.8 & 025 & 26 & 138 & 089 & 179 & $\mathrm{TF}$ \\
\hline 20090809-105559 & 33.05 & 138.19 & 301.8 & 7.1 & 086 & 17 & 168 & 137 & 047 & UF \\
\hline 20090810-195605 & 14.16 & 092.94 & 022.0 & 7.5 & 039 & 36 & -092 & 040 & 130 & NF \\
\hline 20090902-075508 & -08.12 & 107.33 & 053.2 & 7.0 & 054 & 46 & 117 & 126 & 036 & $\mathrm{TF}$ \\
\hline 20090929-174827 & -15.13 & -171.97 & 012.0 & 8.1 & 119 & 38 & -131 & 144 & 054 & NF \\
\hline 20090930-101617 & -00.79 & 099.67 & 077.8 & 7.6 & 074 & 52 & 139 & 133 & 043 & $\mathrm{TF}$ \\
\hline 20091007-220329 & -12.59 & 166.27 & 044.2 & 7.6 & 344 & 41 & 087 & 076 & 166 & TF \\
\hline 20091007-221915 & -11.86 & 166.01 & 041.7 & 7.8 & 337 & 36 & 082 & 072 & 162 & $\mathrm{TF}$ \\
\hline $20091007-231400$ & -13.12 & 166.37 & 042.5 & 7.4 & 341 & 43 & 083 & 076 & 166 & $\mathrm{TF}$ \\
\hline 20091109-104503 & -17.11 & 178.53 & 603.9 & 7.3 & 172 & 42 & 033 & 114 & 024 & $\mathrm{TS}$ \\
\hline 20100103-223642 & -08.88 & 157.21 & 012.0 & 7.1 & 321 & 21 & 102 & 045 & 135 & $\mathrm{TF}$ \\
\hline $20100112-215317$ & 18.61 & -072.62 & 012.0 & 7.0 & 152 & 69 & 159 & 021 & 111 & SS \\
\hline 20100226-203130 & 25.86 & 128.61 & 018.0 & 7.0 & 091 & 80 & 009 & 045 & 135 & SS \\
\hline 20100227-063515 & -35.98 & -073.15 & 023.2 & 8.8 & 019 & 18 & 116 & 095 & 005 & $\mathrm{TF}$ \\
\hline $20100227-080130$ & -38.09 & -075.41 & 019.9 & 7.4 & 003 & 46 & -102 & 011 & 101 & NF \\
\hline $20100311-145536$ & -34.53 & -072.13 & 016.3 & 7.0 & 016 & 06 & -053 & 176 & 086 & UF \\
\hline 20100404-224109 & 32.31 & -115.39 & 012.8 & 7.2 & 223 & 84 & -002 & 178 & 088 & SS \\
\hline 20100406-221519 & 02.07 & 096.74 & 017.6 & 7.8 & 307 & 07 & 88 & 039 & 129 & UF \\
\hline 20100509-055951 & 03.36 & 095.78 & 037.2 & 7.2 & 308 & 15 & 088 & 040 & 130 & $\mathrm{TF}$ \\
\hline $20100527-171455$ & -13.81 & 166.65 & 042.9 & 7.2 & 347 & 44 & 096 & 073 & 163 & $\mathrm{TF}$ \\
\hline 20100612-192700 & 07.85 & 091.65 & 033.1 & 7.5 & 220 & 63 & 031 & 168 & 078 & UF \\
\hline 20100616-031639 & -01.87 & 136.36 & 012.0 & 7.0 & 332 & 80 & -172 & 016 & 106 & SS \\
\hline $20100718-133513$ & -06.22 & 150.33 & 036.7 & 7.3 & 257 & 30 & 082 & 172 & 082 & $\mathrm{TF}$ \\
\hline $20100723-220820$ & 06.54 & 123.59 & 596.8 & 7.3 & 263 & 19 & -031 & 053 & 143 & UF \\
\hline $20100723-225121$ & 06.62 & 123.90 & 576.9 & 7.6 & 233 & 18 & -038 & 026 & 116 & $\mathrm{NF}$ \\
\hline $20100723-231516$ & 06.83 & 123.48 & 641.4 & 7.4 & 254 & 22 & -052 & 054 & 144 & NF \\
\hline $20100810-052354$ & -17.57 & 167.81 & 031.9 & 7.3 & 353 & 33 & 126 & 062 & 152 & $\mathrm{TF}$ \\
\hline $20100812-115121$ & -01.51 & -077.51 & 197.8 & 7.1 & 153 & 21 & -068 & 142 & 052 & $\mathrm{NF}$ \\
\hline 20100903-163558 & -43.56 & 172.12 & 012.0 & 7.0 & 178 & 82 & 001 & 133 & 043 & SS \\
\hline 20100929-171132 & -04.92 & 133.78 & 017.7 & 7.0 & 185 & 43 & -075 & 175 & 085 & NF \\
\hline $20101025-144300$ & -03.71 & 099.32 & 012.0 & 7.8 & 316 & 08 & 096 & 044 & 134 & UF \\
\hline 20101221-171954 & 27.10 & 015.60 & 015.6 & 7.4 & 110 & 40 & -137 & 137 & 047 & $\mathrm{NF}$ \\
\hline $20101225-131651$ & -19.67 & 168.04 & 016.6 & 7.3 & 001 & 48 & -061 & 161 & 071 & NF \\
\hline 20110101-095704 & -27.02 & -063.21 & 586.0 & 7.0 & 360 & 20 & -064 & 166 & 076 & NF \\
\hline 20110102-202027 & -38.71 & -073.84 & 019.4 & 7.1 & 005 & 13 & 097 & 091 & 001 & TF \\
\hline 20110118-202332 & 28.61 & 063.90 & 052.3 & 7.2 & 077 & 31 & -060 & 060 & 150 & $\mathrm{NF}$ \\
\hline 20110309-024532 & 38.56 & 142.78 & 014.1 & 7.3 & 189 & 12 & 078 & 105 & 015 & $\mathrm{TF}$ \\
\hline 20110311-054733 & 37.52 & 143.05 & 020.0 & 9.1 & 203 & 10 & 088 & 115 & 025 & UF \\
\hline 20110311-061559 & 35.92 & 141.38 & 029.0 & 7.9 & 199 & 17 & 084 & 112 & 022 & $\mathrm{TF}$ \\
\hline $20110311-062613$ & 38.27 & 144.63 & 021.1 & 7.6 & 182 & 42 & -100 & 009 & 099 & $\mathrm{NF}$ \\
\hline 20110407-143251 & 38.32 & 141.85 & 053.5 & 7.1 & 019 & 37 & 083 & 113 & 023 & $\mathrm{TF}$ \\
\hline 20110624-030952 & 52.09 & -171.77 & 074.2 & 7.3 & 015 & 10 & -160 & 048 & 138 & $\mathrm{UF}$ \\
\hline 20110706-190333 & -29.22 & -175.83 & 022.3 & 7.6 & 163 & 36 & -114 & 177 & 087 & $\mathrm{NF}$ \\
\hline $20110710-005716$ & 37.98 & 143.33 & 022.0 & 7.0 & 065 & 74 & 016 & 018 & 108 & SS \\
\hline $20110820-165513$ & -18.52 & 167.94 & 033.5 & 7.1 & 342 & 29 & 093 & 070 & 160 & $\mathrm{TF}$ \\
\hline $20110820-181935$ & -18.26 & 167.94 & 036.0 & 7.0 & 343 & 33 & 094 & 070 & 160 & $\mathrm{TF}$ \\
\hline 20110824-174616 & -07.68 & -074.66 & 144.4 & 7.0 & 197 & 40 & -057 & 177 & 087 & $\mathrm{NF}$ \\
\hline $20110903-225546$ & -20.79 & 169.72 & 151.6 & 7.0 & 019 & 29 & 160 & 073 & 163 & UF \\
\hline
\end{tabular}


Table 1. (Continued.)

\begin{tabular}{|c|c|c|c|c|c|c|c|c|c|c|}
\hline \multirow[b]{2}{*}{ Time } & \multicolumn{3}{|c|}{ Location } & \multicolumn{4}{|c|}{ Focal mechanism data } & \multicolumn{2}{|c|}{$\mathrm{SH}$} & \multirow{2}{*}{$\begin{array}{c}\text { Stress } \\
\text { Regime }\end{array}$} \\
\hline & Lat. & Long. & Depth & $M w$ & Strike & Dip & Slip & Max & Min & \\
\hline 20110915-193111 & -21.61 & -179.21 & 624.9 & 7.3 & 312 & 38 & -007 & 091 & 001 & UF \\
\hline 20111021-175728 & -28.83 & -175.72 & 048.4 & 7.4 & 202 & 37 & 083 & 116 & 026 & $\mathrm{TF}$ \\
\hline 20111023-104128 & 38.64 & 043.40 & 012.0 & 7.1 & 246 & 38 & 060 & 174 & 084 & $\mathrm{TF}$ \\
\hline 20111214-050505 & -07.49 & 146.83 & 132.9 & 7.1 & 067 & 24 & -004 & 024 & 114 & UF \\
\hline 20120110-183713 & 02.59 & 092.98 & 023.7 & 7.2 & 103 & 81 & -173 & 147 & 057 & SS \\
\hline 20120202-133449 & -17.69 & 167.11 & 020.5 & 7.0 & 051 & 51 & -043 & 021 & 111 & NF \\
\hline $20120320-180255$ & 16.60 & -098.39 & 015.4 & 7.5 & 296 & 10 & 095 & 024 & 114 & UF \\
\hline $20120325-223721$ & -35.31 & -072.41 & 033.8 & 7.1 & 020 & 16 & 112 & 099 & 009 & $\mathrm{TF}$ \\
\hline 20120411-083931 & 02.35 & 092.82 & 045.6 & 8.6 & 020 & 76 & 005 & 154 & 064 & SS \\
\hline 20120411-104338 & 00.90 & 092.31 & 054.7 & 8.2 & 107 & 83 & -177 & 152 & 062 & SS \\
\hline $20120412-071605$ & 28.57 & -112.76 & 015.8 & 7.0 & 131 & 74 & -177 & 175 & 085 & SS \\
\hline 20120814-025951 & 49.97 & 145.70 & 598.2 & 7.7 & 243 & 63 & 108 & 135 & 045 & $\mathrm{TF}$ \\
\hline $20120827-043740$ & 12.02 & -089.17 & 012.0 & 7.3 & 117 & 76 & 093 & 022 & 112 & $\mathrm{TF}$ \\
\hline $20120831-124744$ & 10.85 & 126.97 & 045.2 & 7.6 & 197 & 51 & 110 & 092 & 002 & $\mathrm{TF}$ \\
\hline $20120905-144223$ & 10.00 & -085.64 & 029.7 & 7.6 & 115 & 73 & 086 & 032 & 122 & $\mathrm{TF}$ \\
\hline $20120930-163145$ & 01.89 & -076.22 & 159.1 & 7.2 & 080 & 54 & -073 & 066 & 156 & NF \\
\hline $20121028-030437$ & 52.61 & -132.06 & 012.0 & 7.8 & 122 & 66 & 083 & 040 & 130 & $\mathrm{TF}$ \\
\hline $20121107-163556$ & 14.11 & -092.43 & 021.3 & 7.4 & 121 & 65 & 094 & 026 & 116 & $\mathrm{TF}$ \\
\hline $20121207-081835$ & 38.01 & 144.09 & 057.8 & 7.2 & 158 & 59 & 048 & 098 & 008 & $\mathrm{TF}$ \\
\hline $20121207-081847$ & 37.77 & 143.83 & 019.5 & 7.2 & 198 & 50 & -090 & 018 & 108 & NF \\
\hline $20121210-165315$ & -06.65 & 129.83 & 159.2 & 7.1 & 048 & 80 & 043 & 179 & 089 & UF \\
\hline $20130105-085832$ & 55.69 & -134.97 & 013.8 & 7.5 & 064 & 89 & 030 & 018 & 108 & UF \\
\hline 20130206-011255 & -11.18 & 165.21 & 020.2 & 7.9 & 142 & 70 & 090 & 052 & 142 & $\mathrm{TF}$ \\
\hline 20130206-012330 & 22.50 & 008.60 & 022.5 & 7.1 & 166 & 52 & -085 & 162 & 072 & NF \\
\hline 20130206-015423 & -10.44 & 165.56 & 014.1 & 7.0 & 160 & 88 & 007 & 115 & 025 & SS \\
\hline $20130208-152654$ & -10.72 & 165.92 & 023.6 & 7.0 & 358 & 85 & 008 & 132 & 042 & SS \\
\hline 20130209-141615 & 01.16 & -077.40 & 152.3 & 7.0 & 265 & 78 & 015 & 038 & 128 & SS \\
\hline 20130406-044242 & -03.33 & 138.49 & 073.6 & 7.0 & 222 & 56 & -043 & 011 & 101 & NS \\
\hline 20130416-104432 & 27.89 & 062.21 & 050.8 & 7.7 & 238 & 56 & -102 & 069 & 159 & NF \\
\hline 20130419-030605 & 46.00 & 150.92 & 104.5 & 7.3 & 244 & 83 & -109 & 099 & 009 & UF \\
\hline $20130523-171913$ & -23.19 & -176.88 & 188.1 & 7.4 & 015 & 82 & -085 & 000 & 090 & UF \\
\hline 20130524-054508 & 54.61 & 153.77 & 611.0 & 8.3 & 012 & 79 & -089 & 009 & 099 & NF \\
\hline $20130707-183542$ & -04.10 & 153.98 & 382.9 & 7.3 & 167 & 47 & -070 & 153 & 063 & NF \\
\hline $20130715-140407$ & -61.05 & -023.51 & 021.5 & 7.3 & 271 & 85 & -018 & 047 & 137 & SS \\
\hline $20130830-162510$ & 51.44 & -175.12 & 026.7 & 7.0 & 064 & 67 & 086 & 159 & 069 & $\mathrm{TF}$ \\
\hline 20130924-113008 & 26.70 & 065.04 & 012.0 & 7.8 & 130 & 87 & 129 & 177 & 087 & UF \\
\hline 20130925-164252 & -16.03 & -074.81 & 046.1 & 7.0 & 133 & 59 & 093 & 040 & 130 & $\mathrm{TF}$ \\
\hline 20131015-001250 & 09.84 & 123.96 & 012.0 & 7.1 & 235 & 50 & 098 & 138 & 048 & $\mathrm{TF}$ \\
\hline 20131025-171025 & 37.17 & 144.66 & 024.9 & 7.1 & 171 & 43 & -107 & 002 & 092 & NF \\
\hline 20131117-090541 & -60.49 & -045.32 & 023.8 & 7.8 & 102 & 44 & 003 & 056 & 146 & UF \\
\hline $20140401-234732$ & -19.70 & -070.81 & 021.6 & 8.1 & 355 & 15 & 106 & 077 & 167 & $\mathrm{TF}$ \\
\hline 20140401-235811 & -19.85 & -071.39 & 020.6 & 7.0 & 177 & 65 & 094 & 082 & 172 & TF \\
\hline $20140403-024336$ & -20.43 & -070.60 & 028.7 & 7.7 & 165 & 76 & 087 & 080 & 170 & $\mathrm{TF}$ \\
\hline 20140411-070734 & -06.78 & 155.09 & 044.1 & 7.1 & 134 & 48 & 093 & 042 & 132 & $\mathrm{TF}$ \\
\hline $20140412-201450$ & -11.35 & 162.24 & 027.3 & 7.6 & 117 & 71 & 028 & 067 & 157 & SS \\
\hline $20140413-123628$ & -11.45 & 161.97 & 037.5 & 7.4 & 289 & 49 & 102 & 010 & 100 & $\mathrm{TF}$ \\
\hline $20140418-142736$ & 17.55 & -101.25 & 018.9 & 7.3 & 115 & 72 & 087 & 029 & 119 & $\mathrm{TF}$ \\
\hline $20140419-132813$ & -06.64 & 154.67 & 036.0 & 7.5 & 135 & 55 & 092 & 044 & 134 & $\mathrm{TF}$ \\
\hline 20140623-205334 & 52.00 & 178.43 & 104.3 & 7.9 & 309 & 84 & -117 & 169 & 079 & UF \\
\hline 20141014-035144 & 12.33 & -088.45 & 040.8 & 7.3 & 309 & 67 & -088 & 127 & 037 & NF \\
\hline $20141101-185730$ & -19.76 & -177.61 & 445.4 & 7.1 & 252 & 80 & 074 & 012 & 102 & $\mathrm{UF}$ \\
\hline
\end{tabular}


Table 1. (Continued.)

\begin{tabular}{|c|c|c|c|c|c|c|c|c|c|c|}
\hline \multirow[b]{2}{*}{ Time } & \multicolumn{3}{|c|}{ Location } & \multicolumn{4}{|c|}{ Focal mechanism data } & \multicolumn{2}{|c|}{$\mathrm{SH}$} & \multirow{2}{*}{$\begin{array}{c}\text { Stress } \\
\text { Regime }\end{array}$} \\
\hline & Lat. & Long. & Depth & $M w$ & Strike & Dip & Slip & Max & Min & \\
\hline 20150213-185928 & 52.70 & -032.74 & 025.2 & 7.1 & 277 & 88 & -170 & 142 & 052 & SS \\
\hline $20150227-134509$ & -07.35 & 122.50 & 551.5 & 7.0 & 137 & 79 & -141 & 178 & 088 & SS \\
\hline 20150329-234855 & -05.18 & 152.59 & 037.6 & 7.5 & 081 & 61 & 90 & 171 & 081 & $\mathrm{TF}$ \\
\hline 20150425-061159 & 27.91 & 085.33 & 012.0 & 7.9 & 101 & 84 & 089 & 016 & 106 & $\mathrm{UF}$ \\
\hline 20150505-014427 & -05.32 & 152.10 & 038.3 & 7.5 & 080 & 58 & 098 & 162 & 072 & $\mathrm{TF}$ \\
\hline $20150507-071034$ & -07.36 & 154.49 & 012.0 & 7.0 & 132 & 51 & -077 & 122 & 032 & NF \\
\hline $20150512-070528$ & 27.67 & 086.08 & 012.0 & 7.2 & 307 & 11 & 117 & 023 & 113 & UF \\
\hline $20150522-214527$ & -10.90 & 163.54 & 015.3 & 7.0 & 252 & 58 & -005 & 028 & 118 & SS \\
\hline 20150530-112311 & 27.94 & 140.56 & 680.7 & 7.9 & 036 & 25 & -039 & 010 & 100 & NF \\
\hline $20150727-214131$ & -02.53 & 138.44 & 040.6 & 7.0 & 327 & 42 & 117 & 040 & 130 & $\mathrm{TF}$ \\
\hline $20150916-225523$ & -31.13 & -072.09 & 017.4 & 8.3 & 007 & 19 & 109 & 087 & 177 & $\mathrm{TF}$ \\
\hline $20150916-231852$ & -31.79 & -071.95 & 035.7 & 7.1 & 349 & 30 & 087 & 081 & 171 & $\mathrm{TF}$ \\
\hline $20151020-215208$ & -14.88 & 167.19 & 140.9 & 7.1 & 189 & 58 & 147 & 064 & 154 & TS \\
\hline 20151026-090947 & 36.55 & 070.42 & 209.4 & 7.5 & 279 & 21 & 085 & 012 & 102 & $\mathrm{TF}$ \\
\hline $20151124-224547$ & -10.73 & -071.12 & 610.7 & 7.5 & 350 & 39 & -080 & 164 & 074 & NF \\
\hline $20151124-225101$ & -10.11 & -071.28 & 627.3 & 7.6 & 350 & 30 & -081 & 164 & 074 & NF \\
\hline 20151204-222507 & -47.81 & 085.14 & 028.4 & 7.1 & 044 & 47 & -054 & 020 & 110 & NF \\
\hline $20151207-075015$ & 38.35 & 072.88 & 012.0 & 7.2 & 303 & 75 & 180 & 168 & 078 & SS \\
\hline 20160124-103037 & 59.75 & -153.27 & 110.7 & 7.1 & 313 & 59 & 152 & 005 & 095 & TS \\
\hline $20160130-032520$ & 53.86 & 158.73 & 168.1 & 7.2 & 324 & 39 & -029 & 111 & 021 & NS \\
\hline $20160302-125005$ & -04.75 & 094.22 & 037.2 & 7.8 & 275 & 87 & 180 & 140 & 050 & SS \\
\hline $20160415-162516$ & 32.84 & 130.77 & 012.9 & 7.0 & 128 & 71 & -014 & 085 & 175 & SS \\
\hline $20160416-235857$ & -00.12 & -080.25 & 022.3 & 7.8 & 027 & 21 & 124 & 100 & 010 & $\mathrm{TF}$ \\
\hline 20160428-193334 & -16.05 & 167.26 & 033.5 & 7.0 & 328 & 36 & 084 & 061 & 151 & TF \\
\hline 20160528-094708 & -56.24 & -026.50 & 068.7 & 7.2 & 223 & 38 & -142 & 071 & 161 & NF \\
\hline 20160729-211834 & 18.50 & 145.70 & 208.9 & 7.7 & 192 & 34 & 132 & 079 & 169 & $\mathrm{TF}$ \\
\hline $20160812-012645$ & -22.56 & 173.07 & 021.8 & 7.2 & 288 & 75 & 176 & 154 & 064 & SS \\
\hline 20160819-073238 & -55.16 & -031.41 & 022.1 & 7.5 & 116 & 24 & 070 & 037 & 127 & $\mathrm{TF}$ \\
\hline 20160829-043018 & 00.13 & -017.72 & 026.8 & 7.1 & 079 & 77 & 176 & 125 & 035 & SS \\
\hline $20160901-163816$ & -37.19 & 179.03 & 027.8 & 7.1 & 354 & 20 & -134 & 017 & 107 & NF \\
\hline 20161113-110353 & -42.03 & 173.85 & 018.8 & 7.8 & 226 & 33 & 141 & 108 & 018 & $\mathrm{TF}$ \\
\hline 20161208-173858 & -10.48 & 161.14 & 046.3 & 7.8 & 151 & 49 & 123 & 038 & 128 & TF \\
\hline $20161217-105155$ & -05.56 & 153.83 & 053.3 & 7.9 & 141 & 36 & 096 & 048 & 138 & $\mathrm{TF}$ \\
\hline $20161225-142237$ & -43.54 & -074.57 & 034.2 & 7.5 & 007 & 21 & 100 & 091 & 001 & $\mathrm{TF}$ \\
\hline
\end{tabular}

Note: The format of time is year/month/day-hour/minute/second. It is considered that positive values indicate north latitude and negative values indicate east longitude respectively in the present study, NF: normal faulting, SS: strike-slip faulting, TF: thrust faulting, NS: intermediate between NF and SS, TS: intermediate between SS and TF and UF: unknown or oblique faulting.

\subsection{Depth}

In seismology, the depth of focus or focal depth refers to the depth at which an earthquake occurs. Earthquakes occurring at a depth of $<70 \mathrm{~km}$ and $>300 \mathrm{~km}$ are classified as shallow-focus and deepfocus earthquakes, respectively, while those with a focal depth between 70 and $300 \mathrm{~km}$ are commonly termed mid-focus earthquakes or intermediate earthquakes (Li 1983; Armstrong et al. 2008). Based on the data in table 1, about $75.8 \%$ of global strong earthquakes are shallow-focus earthquakes (figure 5). Mid- and deep-focus earthquakes only account for $14.7 \%$ and $9.5 \%$, respectively (figure 5).

\subsection{Stress regime}

In the compiled database (table 1), the horizontal stress axes $S_{H \max }$ and $S_{H \min }$ according to Lund and Townend (2007) and stress regime based on the WSM standard were calculated for individual mechanism (figure 2). Generally, the most common stress regime of global strong earthquakes is $\mathrm{TF}$ (47.7\%) (figure 6). 


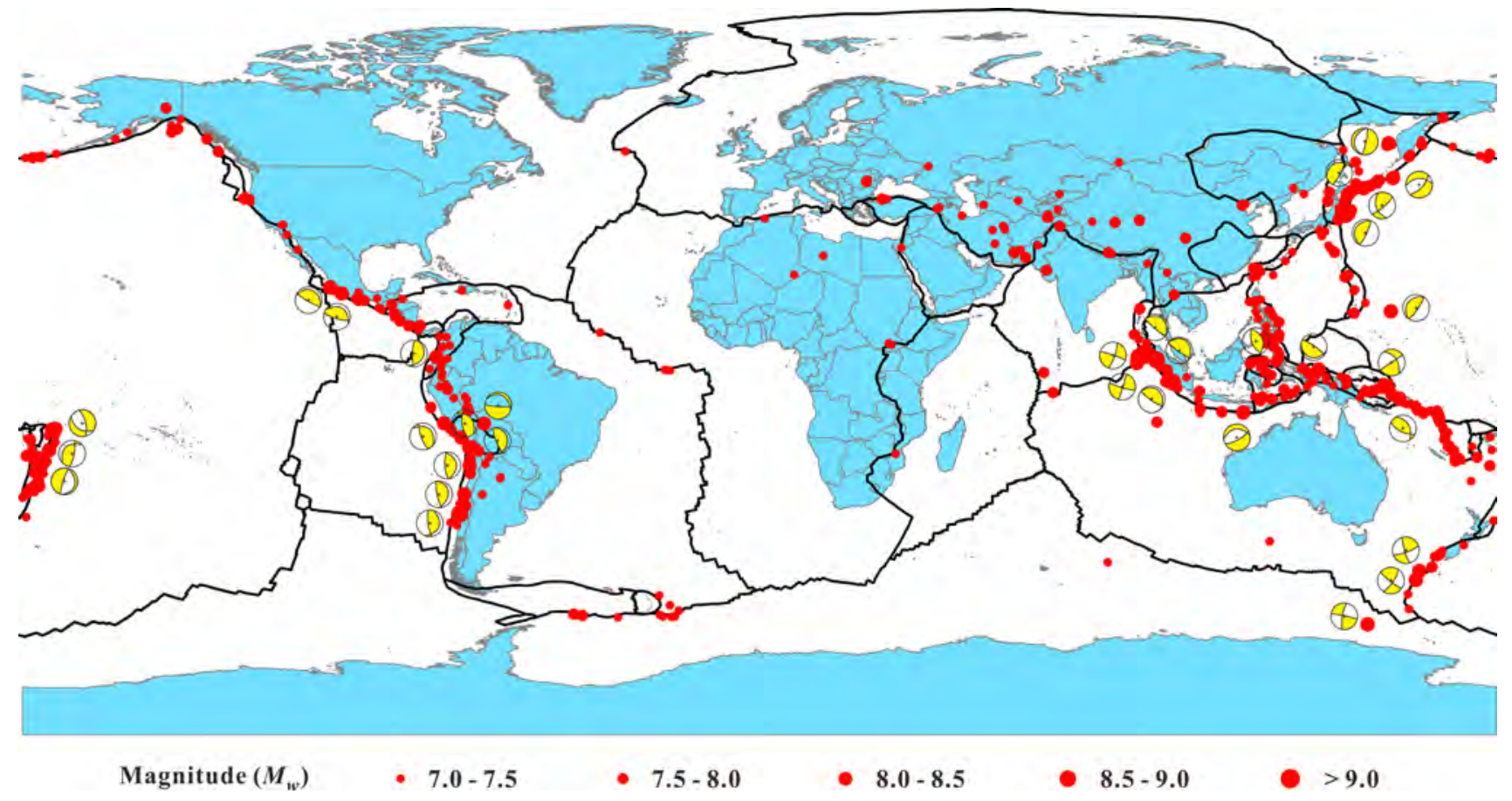

Figure 1. The distribution of global strong earthquakes with focal mechanisms of superstrong earthquakes $(M w \geq 8.0)(1976-$ 2017). Plate boundaries are from Bird (2003).

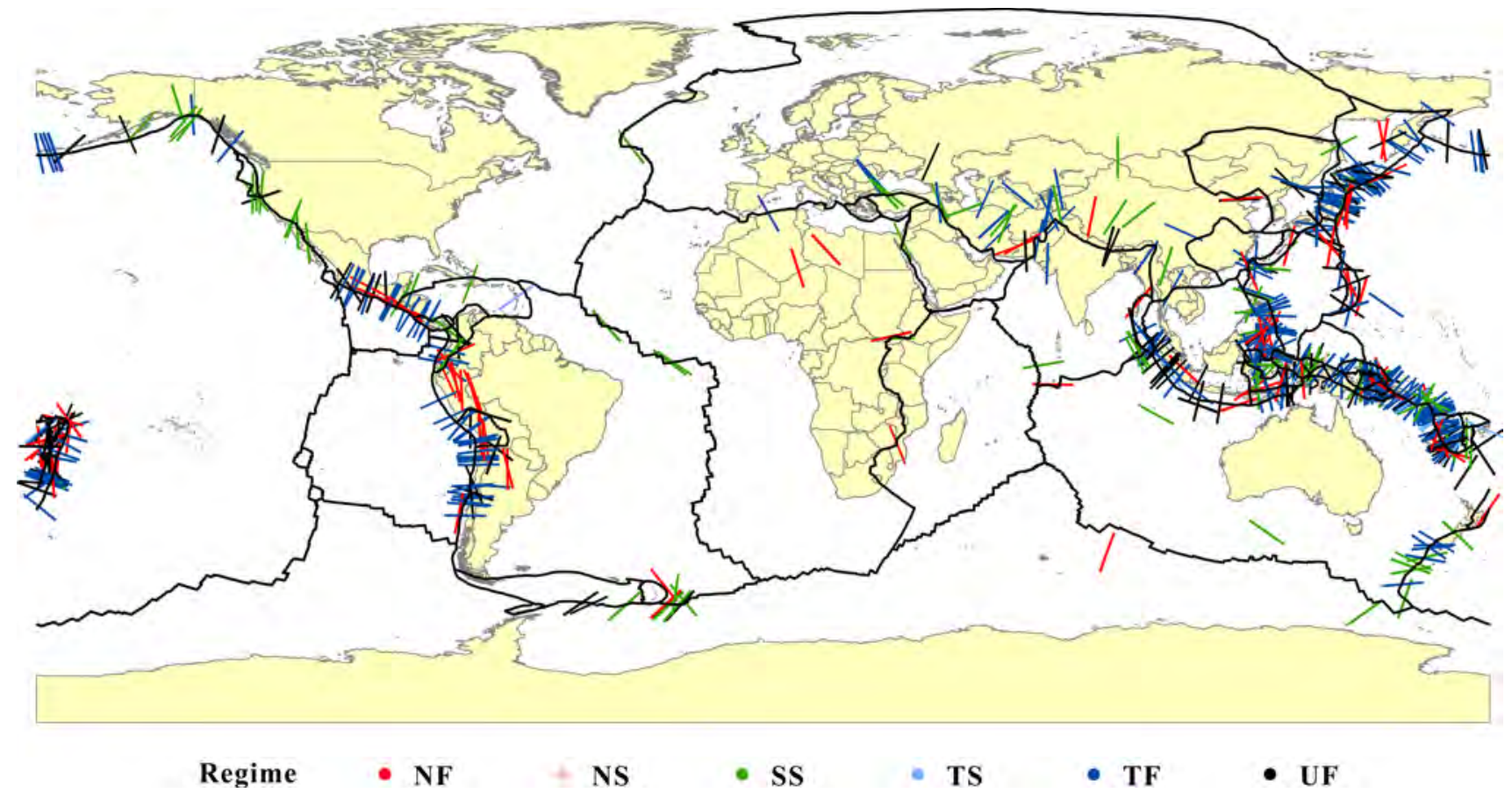

Figure 2. Focal mechanism data of global strong earthquakes (1976-2017) represented as $S_{H \text { max }}$ axes. The extending directions of lines indicate the $S_{H \max }$ axes. NF: normal faulting, SS: strike-slip faulting, TF: thrust faulting, NS: intermediate between NF and SS, TS: intermediate between SS and TF, and UF: unknown or oblique faulting. Plate boundaries are from Bird (2003). 

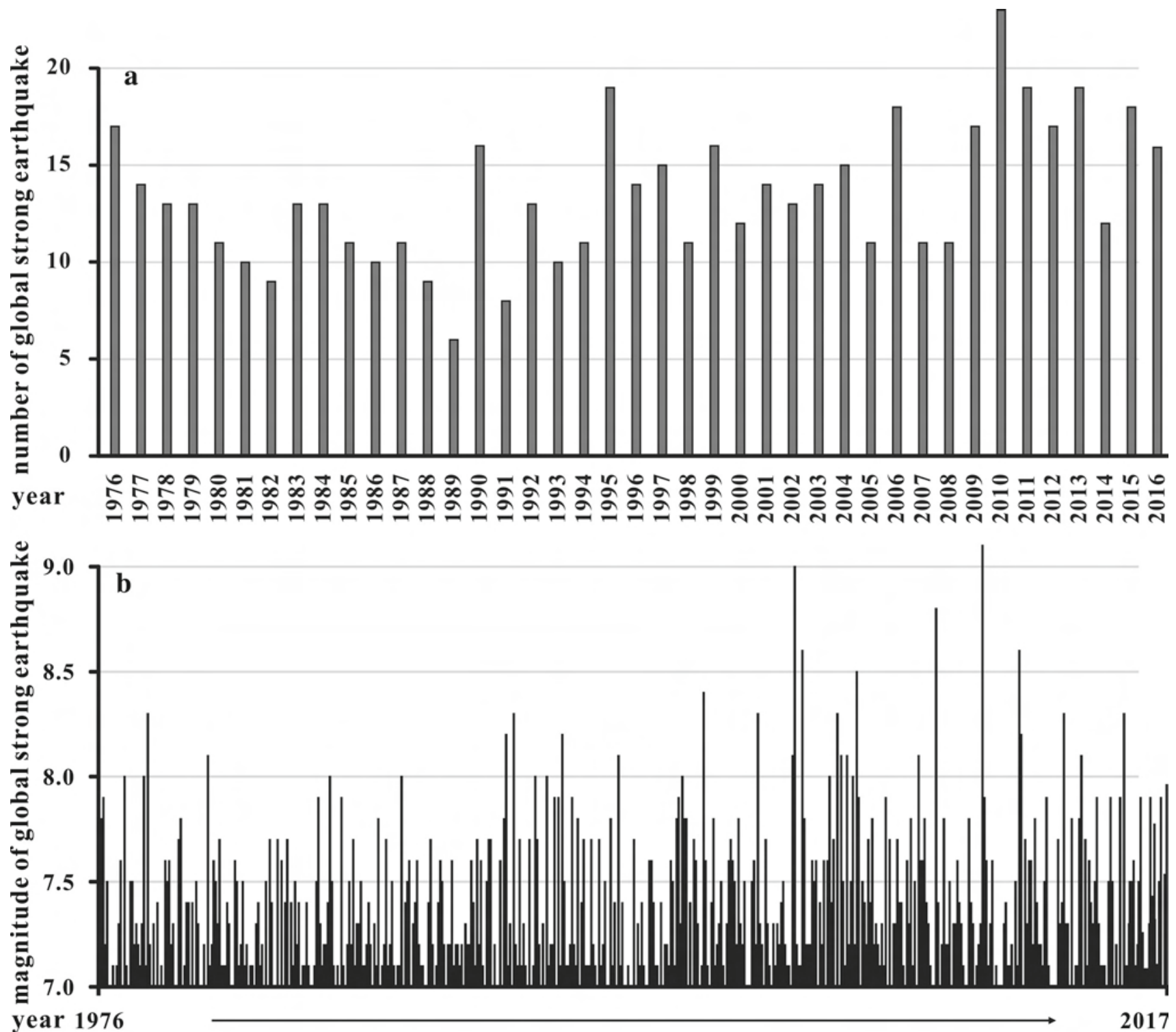

Figure 3. The temporal characteristics of global strong earthquakes (1976-2017). (a) The number of strong earthquakes with time and (b) the magnitude of strong earthquakes with time.

\section{Present-day stress pattern}

\subsection{Zonation}

Since the focal mechanism data are not consistent in terms of stress regime and orientation in the whole world, thus they cannot be inverted altogether (Barth 2007; Delvaux and Barth 2010). Inverting such data together without considering regional deformation characteristics leads to mean deviatoric stresses, which are not representative of the geological reality (Barth 2007; Naimi-Ghassabian et al. 2015). Therefore, in the present study, from a total of 553 data, 518 of them were assembled into 12 sub-areas (Boxes) on the basis of their geographical proximity and tectonic setting to study regional stress regime and changes in stress orientation (figure 7).

Strong earthquakes in western North America continent, resulting from relative movements between Pacific plate and North America plate, are grouped in Box 01. Box 02 is located in the Middle America region, formal stress inversion of strong earthquakes in Box 02 can reveal the relationship among Cocos plate, Caribbean plate, Pacific plate, North America plate and Nazca plate. The dominant NF- and TF-regime strong earthquakes in western South America continent are grouped in Box 03 and Box 04, respectively, which indicate relative movements between Nazca plate and 


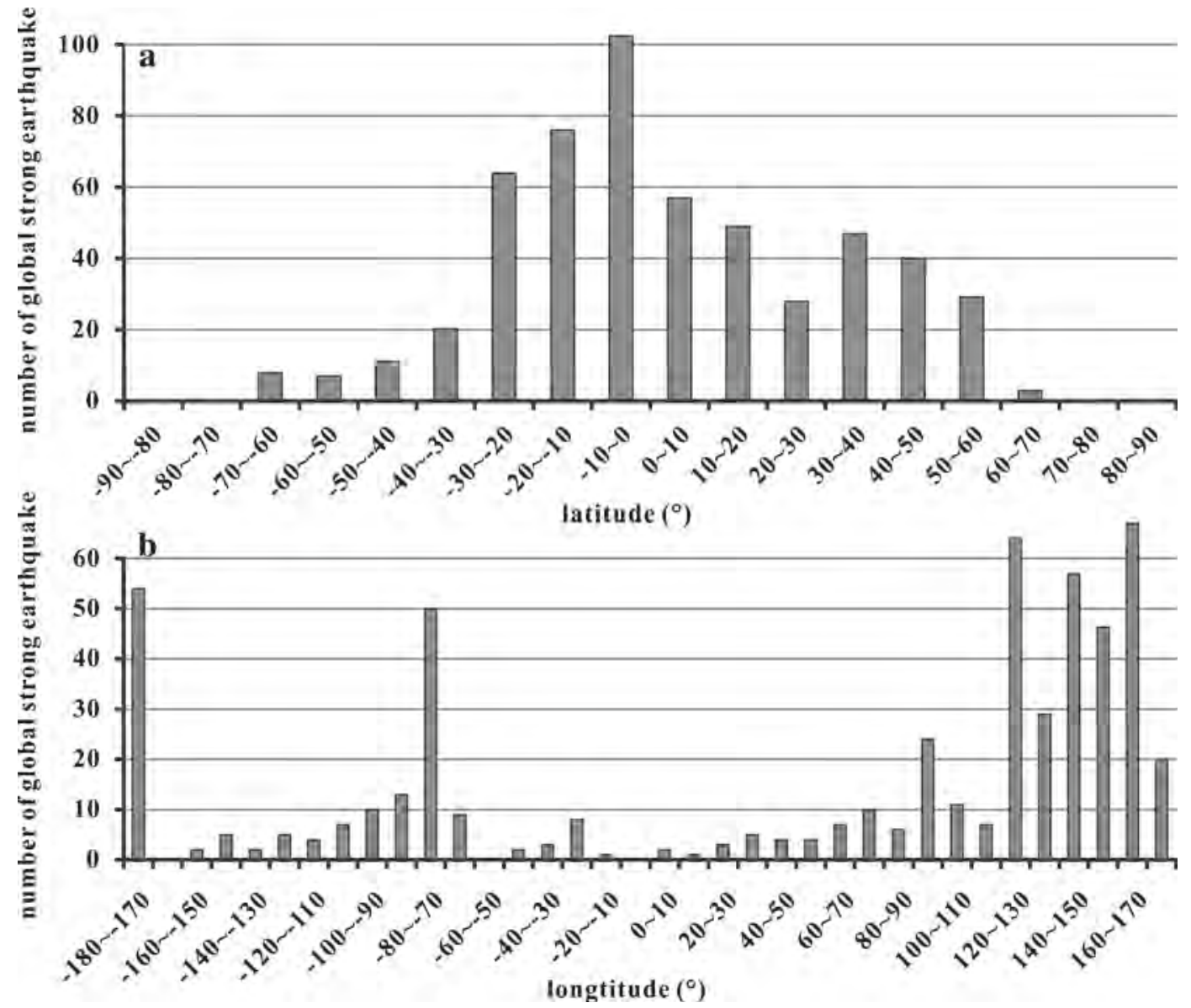

Figure 4. The spatial characteristics of global strong earthquakes (1976-2017) (a) distribution along latitude and (b) distribution along longitude.

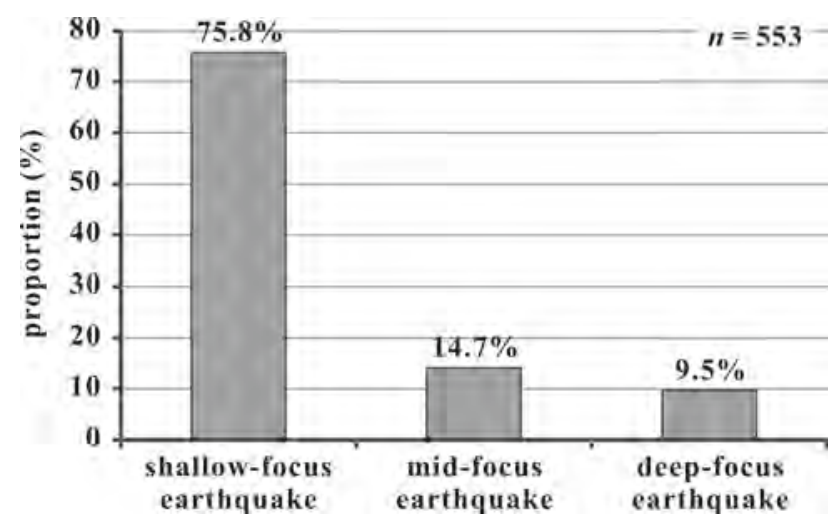

Figure 5. The depths of global strong earthquakes (19762017).

South America plate. Formal stress inversion of strong earthquakes in Box 05 can reveal the relationship among South America plate, Antarctica plate, Sandwich plate and Scotia plate. Boxes 06, 07 and 08 are located in eastern Australia continent, strong earthquakes happened there will

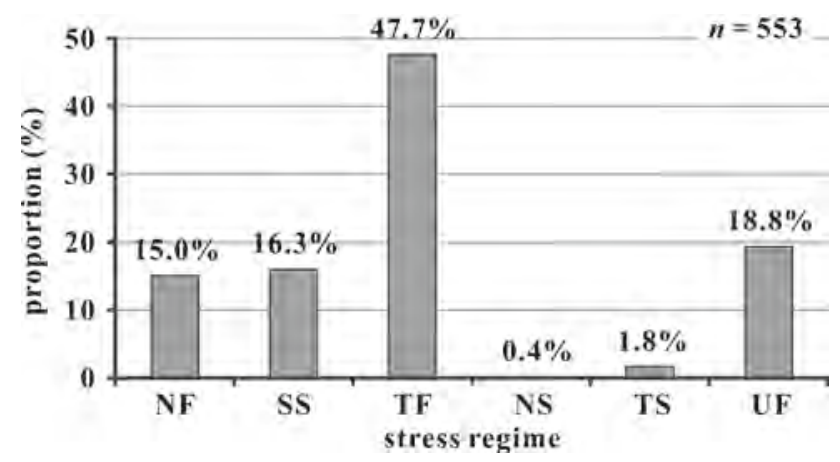

Figure 6. The stress regimes of global strong earthquakes (1976-2017). NF: normal faulting, SS: strike-slip faulting, TF: thrust faulting, NS: intermediate between NF and SS, TS: intermediate between SS and TF, and UF: unknown or oblique faulting.

indicate relative movements between Pacific plate and Australia plate. Inversion of strong earthquakes in Box 09 indicate the stress state caused by Pacific plate, Okhotsk plate and Philippine Sea plate. Strong earthquakes happened in southeastern Asia are grouped in Box 10, which result from 


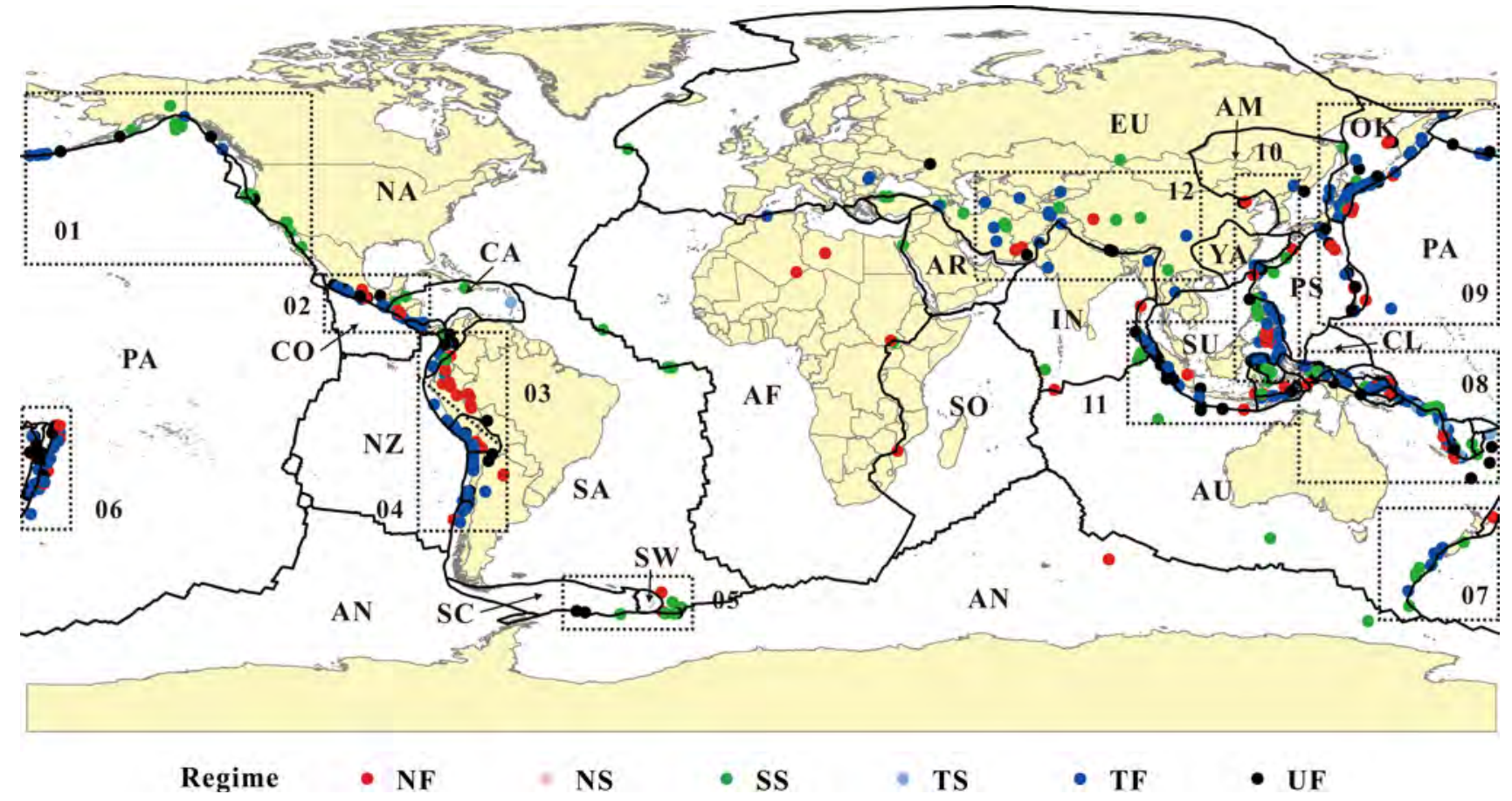

Figure 7. Focal mechanism data of global strong earthquakes (1976-2017) assembled into Boxes for stress inversion. Plate boundaries are from Bird (2003). The main global plates are shown and listed with two-letter plate identifiers - AF: Africa plate, AM: Amur plate, AN: Antarctica plate, AR: Arabia plate, AU: Australia plate, CA: Caribbean plate, CL: Caroline plate, CO: Cocos plate, EU: Eurasia plate, IN: India plate, NA: North America plate, NZ: Nazca plate, OK: Okhotsk plate, PA: Pacific plate, PS: Philippine Sea plate, SA: South America plate, SC: Scotia plate, SO: Somalia plate, SU: Sunda plate, SW: Sandwich plate, and YA: Yangtze plate.

relative movements among Amur plate, Philippine Sea plate and Yangtze plate. Box 11 is located in northwestern Australia continent, inversion of strong earthquakes in Box 11 can reveal the relationship between Sunda plate and Australia plate. Box 12 in located in the Eurasia continent, strong earthquakes in Box 12 mainly result from relative movements between India plate and Eurasia plate (figure 7).

\subsection{Methodology}

Many researchers have attempted to estimate tectonic stresses using a wide variety of direct inversion, iterative and grid search methods adapted for the reconstruction of past and present-day stresses from fault kinematics and/or earthquake focal mechanical data (e.g., Angelier 1979, 1994; Etchecopar et al. 1981; Gephart and Forsyth 1984; Delvaux et al. 1995, 1997; Delvaux and Barth 2010; Ju et al. 2012; Hussein et al. 2013; NaimiGhassabian et al. 2015).

Generally, formal stress inversion of focal mechanism data is based on two assumptions (Bott 1959; Carey-Gailhardis and Vergely 1992; Delvaux et al.
1997; Delvaux and Barth 2010): (a) the stress field is uniform and invariant in space and time, and (b) earthquake slip occurs in the direction of maximum shear stress (Wallace-Bott hypothesis).

In the present study, formal stress inversions were performed to study the global present-day stress field with the Rotational Optimization Method (ROM) (Delvaux and Sperner 2003), which can give four parameters of the reduced stress tensor that best model the stress state in a given region: the orientation of three principal stress axes $\left(\sigma_{1}, \sigma_{2}\right.$ and $\left.\sigma_{3} ; \sigma_{1} \geq \sigma_{2} \geq \sigma_{3}\right)$ and the stress ratio $R$, which shows the magnitude of $\sigma_{2}$ relative to the magnitudes of $\sigma_{1}$ and $\sigma_{3}$ (Delvaux and Sperner 2003; Cortes and Angelier 2005; Delvaux and Barth 2010).

$$
R=\frac{\sigma_{2}-\sigma_{3}}{\sigma_{1}-\sigma_{3}} .
$$

The ROM is a new iterative inversion procedure presented by Delvaux and Sperner (2003). In this method, both nodal planes of each focal mechanism are compared to a stress tensor, and the plane that has the smaller value of the misfit will be considered as the actual fault plane. The selected fault 
Table 2. Stress inversion results for the 12 Boxes (parameters for stress map).

\begin{tabular}{|c|c|c|c|c|c|c|c|c|c|c|c|c|c|c|}
\hline \multicolumn{2}{|c|}{ Box definition } & \multicolumn{7}{|c|}{ Reduced stress tensor parameters } & \multicolumn{2}{|c|}{$\mathrm{SH}$} & \multicolumn{4}{|c|}{ Misfit, regime and quality rank } \\
\hline No. & Data & $\sigma_{1} \mathrm{pl}$ & $\sigma_{1} \mathrm{az}$ & $\sigma_{2} \mathrm{pl}$ & $\sigma_{2} \mathrm{az}$ & $\sigma_{3} \mathrm{pl}$ & $\sigma_{3} \mathrm{az}$ & $R$ & Max & Min & Misfit(avg.) & $R^{\prime}$ & Regime & $Q$ \\
\hline 01 & 025 & 15 & 187 & 17 & 092 & 67 & 317 & 0.17 & 008 & 098 & 14.7 & 2.17 & $\mathrm{TF}$ & $\mathrm{B}$ \\
\hline 02 & 032 & 41 & 207 & 48 & 012 & 08 & 111 & 0.14 & 026 & 116 & 05.6 & 1.86 & NS & A \\
\hline 03 & 018 & 65 & 255 & 11 & 010 & 22 & 105 & 0.03 & 071 & 161 & 07.8 & 0.03 & $\mathrm{NF}$ & A \\
\hline 04 & 025 & 10 & 083 & 03 & 174 & 79 & 278 & 0.83 & 080 & 170 & 13.2 & 2.83 & $\mathrm{TF}$ & A \\
\hline 05 & 012 & 84 & 224 & 06 & 050 & 01 & 320 & 0.90 & 050 & 140 & 15.7 & 0.90 & $\mathrm{NF}$ & B \\
\hline 06 & 047 & 23 & 099 & 30 & 355 & 51 & 220 & 0.17 & 101 & 191 & 09.3 & 2.17 & UF & B \\
\hline 07 & 011 & 12 & 277 & 03 & 186 & 78 & 084 & 0.36 & 097 & 187 & 13.0 & 2.36 & $\mathrm{TF}$ & B \\
\hline 08 & 129 & 06 & 217 & 02 & 127 & 83 & 019 & 0.69 & 038 & 128 & 14.0 & 2.69 & $\mathrm{TF}$ & B \\
\hline 09 & 080 & 14 & 143 & 09 & 235 & 73 & 357 & 0.80 & 133 & 223 & 15.3 & 2.80 & $\mathrm{TF}$ & B \\
\hline 10 & 058 & 02 & 259 & 22 & 350 & 68 & 164 & 0.71 & 078 & 168 & 15.0 & 2.71 & $\mathrm{TF}$ & B \\
\hline 11 & 055 & 13 & 190 & 07 & 282 & 76 & 041 & 0.61 & 007 & 097 & 16.4 & 2.61 & $\mathrm{TF}$ & B \\
\hline 12 & 026 & 28 & 197 & 21 & 095 & 53 & 333 & 0.14 & 019 & 109 & 09.6 & 2.14 & $\mathrm{TF}$ & B \\
\hline
\end{tabular}

Note: (1) box definition: Box number and the number of data in the Box. (2) Reduced stress tensor parameters: plunge (pl) and azimuth (az) of the principal stress axes $\left(\sigma_{1}, \sigma_{2}\right.$ and $\left.\sigma_{3}\right)$ and stress ratio $R$. (3) SH: $S_{H \max }$ and $S_{H \min }$. (4) Misfit, regime and quality rank: average misfit angle, stress regime index, regime based on the WSM standard, and quality rank $(Q)$. NF: normal faulting, SS: strike-slip faulting, TF: thrust faulting, NS: intermediate between NF and SS, TS: intermediate between SS and TF, and UF: unknown or oblique faulting.

planes are then inverted to calculate the principal stress axes and stress ratio (Gephart and Forsyth 1984; Delvaux and Barth 2010; Naimi-Ghassabian et al. 2015).

To perform the inversion, the Win-Tensor program (Delvaux and Sperner 2003) was used. First, the range of possible orientations $\sigma_{1}$ and $\sigma_{3}$ was determined based on the "Right Dihedron Method (RDM)", which is independent from the choice of the nodal planes (Angelier 1979, 1994) and can make a rough estimation of the four parameters of the reduced stress tensor. It allows us not only to obtain the first estimation of the stress ratio, but also to filter out the focal mechanisms that are not compatible with the dominant dataset (Hussein et al. 2013). The initial result is used as a starting point for the ROM using the misfit function F5 in the program. The function F5 varies from 0 (perfect fit) to 360 (perfect misfit) and is independent from the dimensions of the stress ellipsoid.

In general, the stress regime can be expressed with the Stress Regime Index (SRI, $R^{\prime}$ ), which is calculated from the stress ratio $(R$, equation 1$)$. The $R^{\prime}$ is defined as (Delvaux et al. 1995, 1997; Delvaux and Barth 2010):

$\left(R^{\prime}=R\right)$ for normal faulting regimes (NF),

$\left(R^{\prime}=2-R\right)$ for strike-slip regimes (SS), and

$\left(R^{\prime}=2+R\right)$ for thrust faulting regimes (TF).
Based on the definition of $R^{\prime}$, a continuous scale is formed: 0 to 1 for NF, 1 to 2 for SS, and 2 to 3 for TF.

The quality evaluation was based on the updated quality ranking system of the WSM (Heidbach et al. 2010). Each stress data record is assigned a quality between $\mathrm{A}$ and $\mathrm{E}$ with $\mathrm{A}$ being the highest quality. A-quality means that the orientation of the maximum horizontal compressional stress $S_{H}$ is accurate to within $\pm 15^{\circ}$, B-quality to within $\pm 15^{\circ}-20^{\circ}$, C-quality to within $\pm 20^{\circ}-25^{\circ}$, D-quality to within $\pm 20^{\circ}-40^{\circ}$, and E-quality data records have standard deviations greater than $40^{\circ}$ or do not provide sufficient information. In general, A-, Band C-quality stress indicators are considered reliable for the use in analyzing stress patterns and the interpretation of geodynamic processes (Delvaux and Barth 2010; Naimi-Ghassabian et al. 2015; Soumaya et al. 2015).

In the present study, the best-fit stress tensor on both nodal planes was determined with the ROM first, and was then applied on all focal planes and considered as the actual fault plane. The data were filtered in order to obtain good quality tensors, to keep the most compatible focal plane for each mechanism and eventually to remove those mechanisms for which both focal planes gave a larger angular misfit until all misfit angles fall below $30^{\circ}-40^{\circ}$. 

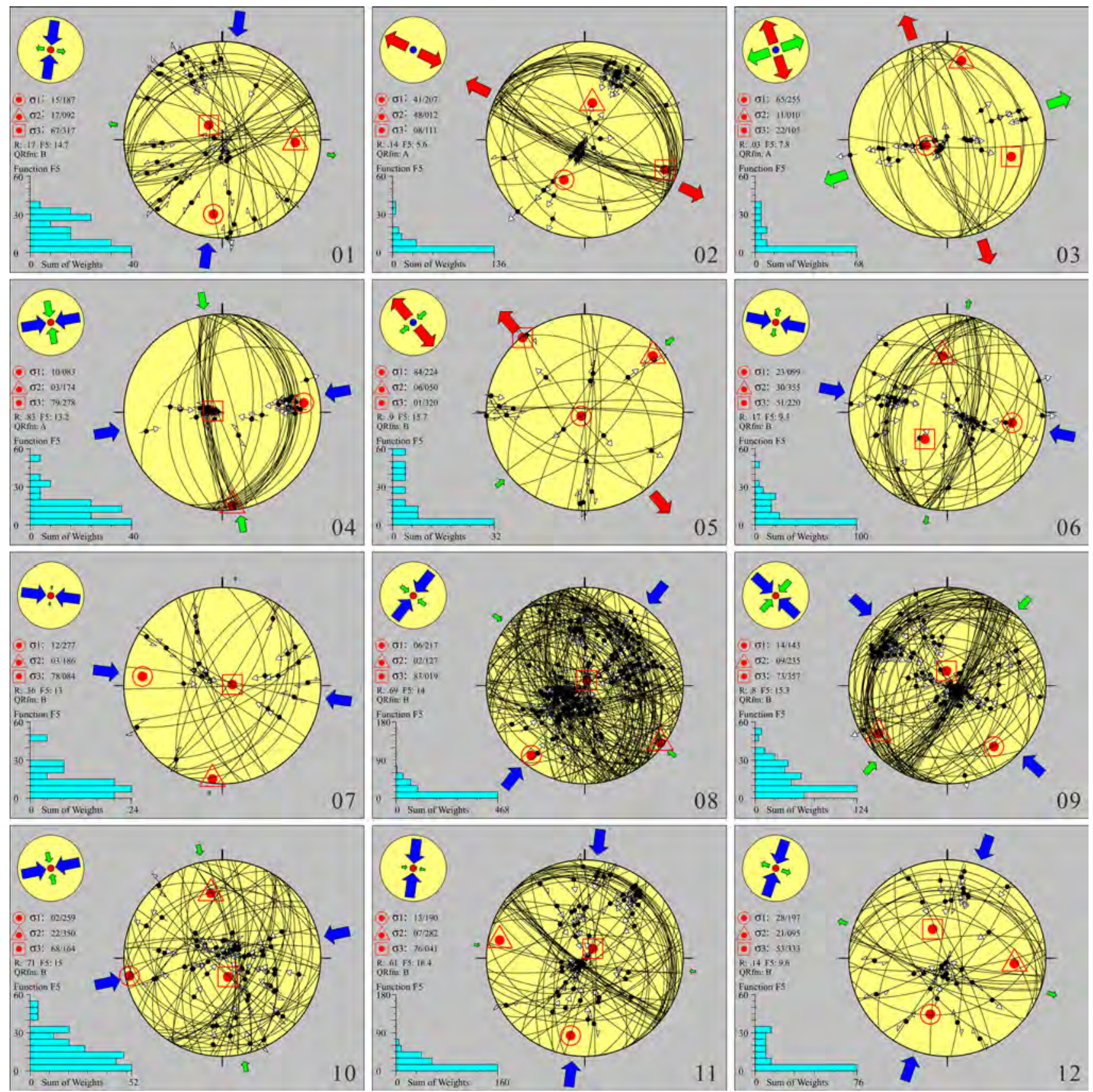

Figure 8. Stress inversion and focal plane selection results. Lower-hemisphere equal-area stereo-plots of the selected focal planes and associated slip lines. The related $S_{H \max }$ and $S_{H \min }$ orientations are represented by large arrows outside the stereogram. Their length and colour symbolize the horizontal deviatoric stress magnitude. Red outward arrows: $\sigma_{3}$ stress axis, green arrows: $\sigma_{2}$ stress axis and blue inward arrows: $\sigma_{1}$ stress axis. The vertical stress is symbolized in the small circle on the upper left corner by a solid circle, red for compressional regimes, green for strike-slip regimes and blue for extensional regimes. Stress inversion results are represented by the orientation of the three principal stress axes (a dot surrounded by a circle for $\sigma_{1}$, a triangle for $\sigma_{2}$, and a square for $\sigma_{3}$ ). The histogram on the lower left corner of the figures represents the distribution of the misfit angle, weighted arithmetically based on the magnitude.

\subsection{Results}

The stress regime in western North America continent is TF $\left(R^{\prime}=2.17\right.$, Box 01) with a $\sim \mathrm{N}-\mathrm{S}-$ trending $\left(\mathrm{N} 008^{\circ} \mathrm{E}\right) S_{H \max }$ axis. The stress tensor in western Middle America region is NS $\left(R^{\prime}=1.86\right.$, Box 02) regime with a NNE-SSW-trending $\left(\mathrm{N} 026^{\circ} \mathrm{E}\right) S_{H \max }$ axis. The northwestern and southwestern South America continent indicate a NF $\left(R^{\prime}=0.03\right.$, Box 03) and TF $\left(R^{\prime}=2.83\right.$, Box 04) 


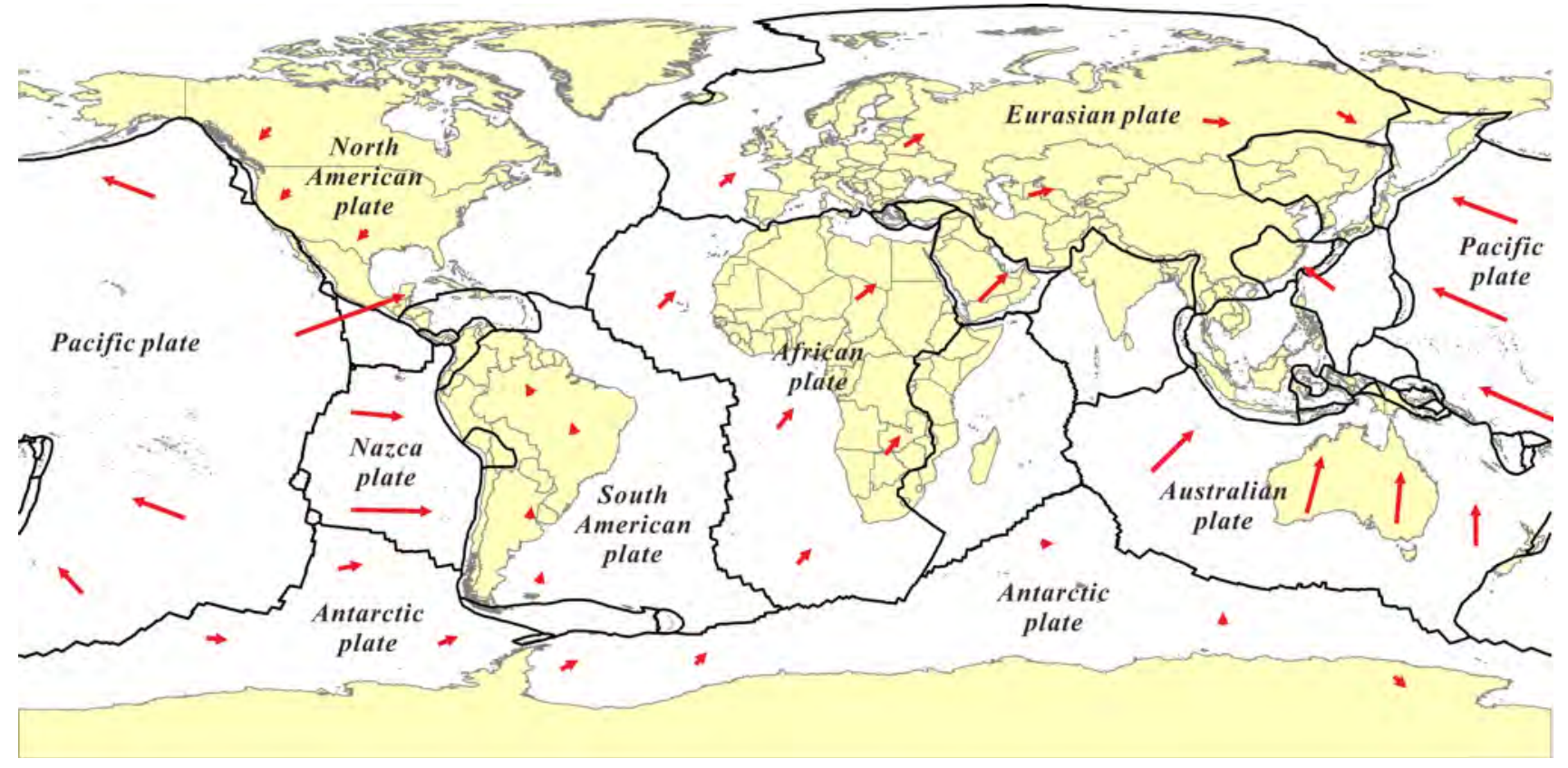

Figure 9. Tectonic maps showing the velocities for major plates in the no-net-rotation reference frame (after Kreemer et al. 2003). Plate boundaries are from Bird (2003).

regime, respectively. The $S_{H \max }$ axis of Box 03 is NEE-SWW-trending $\left(\mathrm{N} 071^{\circ} \mathrm{E}\right)$ and NEE-SWWtrending $\left(\mathrm{N} 080^{\circ} \mathrm{E}\right)$ in Box 04 . The stress regime in southeastern South America continent is NF with a $R^{\prime}$ of 0.90 (Box 05), and its $S_{H \max }$ axis is NESW-trending $\left(\mathrm{N} 040^{\circ} \mathrm{E}\right)$ (table 2 and figure 8 ).

Boxes 06-08 are located in the east of Australia continent (figure 7). The stress tensor indicate a UF $\left(R^{\prime}=2.17\right.$, Box 06; eastern Australia), TF $\left(R^{\prime}=2.36\right.$, Box 07; southeastern Australia), and TF $\left(R^{\prime}=2.69\right.$, Box 08; northeastern Australia) regime, respectively. The $S_{H \max }$ axis is in the

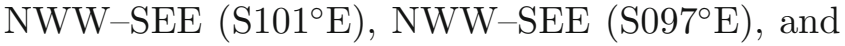
$\mathrm{NE}-\mathrm{SW}\left(\mathrm{N} 038^{\circ} \mathrm{E}\right)$ direction, respectively (table 2 and figure 8).

All the stress regimes in eastern, southeastern, southern and central Asia indicate TF $\left(R^{\prime}=2.80\right.$, Box 09; $R^{\prime}=2.71$, Box 10; $R^{\prime}=2.61$, Box 11 and $R^{\prime}=2.14$, Box 12). The $S_{H \max }$ axis is $\mathrm{NW}-\mathrm{SE}$-trending $\left(\mathrm{S} 133^{\circ} \mathrm{E}\right)$ for eastern Asia, NEE-SWW-trending $\left(\mathrm{N} 078^{\circ} \mathrm{E}\right)$ for southeastern Asia, $\sim \mathrm{N}-\mathrm{S}$-trending $\left(\mathrm{N} 007^{\circ} \mathrm{E}\right)$ for southern Asia, and NNE-SSW-trending $\left(\mathrm{N} 019^{\circ} \mathrm{E}\right)$ for central Asia (table 2 and figure 8 ).

\section{Discussions}

Plate motions provide first order insights into the interplay between the lithosphere and the mantle, which can be determined using seafloor magnetic anomalies, fracture zones and transform fault strikes and earthquakes slip vectors (Coltice et al. 2017). Therefore, the present study results combined with the velocities of major global plates (in the no-net-rotation reference frame; Kreemer et al. 2003) provided new insights into global tectonics and stress pattern (figure 9). The North American and Eurasian plate move to west and east, respectively, with the North Atlantic Ridge as their boundary. The African plate moves towards northeast with an average velocity of about $3.0 \mathrm{~m} / \mathrm{yr}$. The movements of the Australian and Pacific plate point to north and northwest, respectively, with an average velocity of about $6.0 \mathrm{~cm} / \mathrm{yr}$ and $7.0 \mathrm{~cm} / \mathrm{yr}$. Different velocities of global plates and their movement directions cause the difference in stress pattern and tectonics. Heidbach et al. (2010) suggest that tectonic stress field can be classified as a function of the spatial scale of investigation. The 1st order stress field is of continental scale and from the results of forces generated at plate boundaries, most importantly mid-ocean ridge 'push', subduction slab 'pull', trench 'suction' and resistance due to continental collision (Zoback 1992; Reynolds et al. 2002; Delvaux and Barth 2010; Tingay et al. 2010). Plates bordered by oceanic trenches move $\sim 8$ times faster than those bordered by spreading ridges, which supports the process of subduction largely dominates others in its ability to move the plates (Coltice et al. 2017). 
As global strong earthquakes are primarily located in regions of plate boundaries (figure 1), the stress inversion in the present study reveals the present-day 1st order stress field and regime. In addition, the results suggest that different regions (Boxes) have different tectonic stress regimes (figure 8 and table 2), which agree well with the present global tectonic framework. The distribution of global strong earthquakes also suggests that it has a relationship with the earth rotation rate. Larger rotation rate indicates more strong earthquakes (Tang 1997). Besides, Ma et al. (2007) indicated that the number of strong earthquakes (especially the shallow-focus ones) is highly related to the Earth variable rotation rate.

Modeling of present-day plate motions indicated that the ratio between toroidal and poloidal velocity was about 0.32 (Lithgow-Bertelloni and Richards 1998), which suggests that more TFand NF-type earthquakes happened in the lithosphere ( $\mathrm{Yu}$ et al. 2003). In fact, most of the seismic energy was released by TF- and NF-type earthquakes resulting from plate movements $(\mathrm{Yu}$ et al. 2003), especially the TF-type. Generally, the TF-type strong earthquakes are mainly distributed on the circum-pacific seismic belt and the Himalaya collision boundary as shown in Boxes of 01, 04, 06-12 (figures 2, 8 and table 2). The NFtype strong earthquakes occurred in zones of the ocean ridge and continental rift valleys (figures 2 and 8). The toroidal to poloidal ratio of mantle convection determines the release of seismic energy, and the heterogeneous mantle motion act as an important factor for the distribution of different types of strong earthquakes in the world ( $\mathrm{Yu}$ et al. 2003; Martin et al. 2008; Hong et al. 2009).

\section{Conclusions}

Earthquakes occurred on the Earth can serve as important sources for describing the present-day tectonic stress field and regime. In the present study, altogether 553 focal mechanism data from the year 1976 to 2017 with $M w \geq 7.0$ were compiled in the Global/Harvard CMT catalogue. The characteristics of global strong earthquakes and present-day stress pattern were analyzed based on the database. The world has stepped into a natural disaster-prone period with frequent strong earthquakes, revealing the temporal and spatial changes in stress field and geodynamic regime. Global strong earthquakes are primarily distributed around plate boundaries, and $75.8 \%$ of them are shallow-focus earthquakes. The most common stress regime of global strong earthquakes is thrust faulting (TF). We grouped 518 of global strong earthquakes into 12 regions (Boxes) based on their geographical proximity and tectonic setting. For each box, the present-day stress field and regime have been obtained by formal stress inversion. Generally, the maximum horizontal principal stress direction was $\sim \mathrm{N}$-S-trending in western North America and southwestern Indonesia, NNE-SSW-trending in western Middle America and central Asia, $\sim \mathrm{NE}-$ SW in southeastern South America and northeastern Australia, NEE-SWW-trending in western South America and southeastern Asia, $\sim \mathrm{E}-\mathrm{W}$-trending in southeastern Australia, and $\sim \mathrm{NW}-\mathrm{SE}$-trending in eastern Asia. The division and distribution of global plates, the Earth's rotation rate and the toroidal to poloidal ratio of mantle convection influence the global present-day stress pattern.

\section{Acknowledgements}

We would like to express our gratitude to the anonymous reviewers for giving constructive suggestions and comments which improved this manuscript in many aspects. Many thanks to the Global CMT Project for the earthquake data. The work was financially supported by the Key Laboratory of Coal-based $\mathrm{CO}_{2}$ Capture and Geological Storage, Jiangsu Province (China University of Mining and Technology 2016B04), China Postdoctoral Science Foundation (2015M581891), Postdoctoral Science Foundation of Jiangsu Province (1501059A) and the Priority Academic Program Development of Jiangsu Higher Education Institutions.

\section{References}

Angelier J 1979 Determination of the mean principal directions of stresses for a given fault population; Tectonophys. 56 T17-T26.

Angelier J 1994 Fault slip analysis and paleostress reconstruction; In: Continental Deformation (ed.) Hancock P L, Pergamon, Oxford, pp. 101-120.

Armstrong D, Mugglestone F, Richards R and Stratton F 2008 OCR Geology AS \&3 A2 Student Book: Exclusively Endorsed by OCR for GCE Geology; Pearson Education Limited, 336p.

Barth A 2007 Frequency sensitive moment tensor inversion for light to moderate magnitude earthquake in Eastern 
Africa and derivation of the regional stress field; $\mathrm{PhD}$ thesis, University of Karlsruhe.

Bird P 2003 An updated digital model of plate boundaries; Geochem. Geophys. Geosyst. 4(3) 1-52.

Bott M H P 1959 The mechanisms of oblique slip faulting; Geol. Mag. 96 109-117.

Carey-Gailhardis E and Vergely P 1992 Graphical analysis of fault kinematics and focal mechanisms of earthquakes in term of stress: The right dihedral method, use and pitfalls; Ann. Tecton. VI(1) 3-9.

Coltice N, Gerault M and Ulvrova M 2017 A mantle convection perspective on global tectonics; Earth Sci. Rev. 165 $120-150$.

Cortes M and Angelier J 2005 Current states of stress in the northern Andes as indicated by focal mechanisms of earthquakes; Tectonophys. 403 29-58.

Delvaux D and Barth A 2010 African stress pattern from formal inversion of focal mechanism data; Tectonophys. $482105-128$.

Delvaux D, Moeys R, Stapel G, Melnikov A and Ermikov V 1995 Palaeostress reconstruction and geodynamics of the Baikal region, Central Asia. Part 1: Palaeozoic and Mesozoic pre-rift evolution; Tectonophys. 252 61-101.

Delvaux D, Moeys R, Stapel G, Petit C, Levi K, Miroshnichenko A, Ruzhich V and Sankov V 1997 Palaeostress reconstruction and geodynamics of the Baikal region, Central Asia. Part 2: Cenozoic rifting; Tectonophys. 282 $1-38$.

Delvaux D and Sperner B 2003 Stress tensor inversion from fault kinematic indicators and focal mechanism data: The TENSOR program; In: New Insights into Structural Interpretation and Modeling (ed.) Nieuwland D A, Geol. Soc. London, Spec. Publ. 212 75-100.

Ding Z Y, Qian X L, Huo H and Yang Y Q 1998 A new method for quantitative prediction of tectonic fractures - Two Factor Method; Oil Gas Geol. 19(1) 1-7, 14 (in Chinese with English abstract).

Dziewonski A M, Chou T A and Woodhouse J H 1981 Determination of earthquake source parameters from waveform data for studies of global and regional seismicity; J. Geophys. Res. 86 2825-2852.

Dziewonski A M, Ekstrom G, Franzen J E and Woodhouse J H 1987 Global seismicity of 1977: Centroid-moment tensor solutions for 471 earthquakes; Phys. Earth Planet. In. 45 11-36.

Etchecopar A, Vasseur G and Daignieres M 1981 An inverse problem in microtectonics for the determination of stress tensors from fault striation analysis; J. Struct. Geol. 3 51-65.

Fuchs K and Muller B 2001 World Stress Map of the Earth: A key to tectonic processes and technological applications; Sci. Nat. 88(9) 357-371.

Frisch W, Meschede M and Blakey R 2011 Plate Tectonics: Continental Drift and Mountain Building, SpringerVerlag, Heidelberg, 212p.

Gephart J W and Forsyth D W 1984 An improved method for determining the regional stress tensor using earthquake focal mechanism data: Application to the San Fernando earthquake sequence; J. Geophys. Res. 89(B11) 9305-9320.

Heidbach O, Tingay M, Barth A, Reinecker J, Kurfeß D and Muller B 2010 Global crustal stress pattern based on the World Stress Map database release 2008; Tectonophys. $4823-15$.

Hong Z J, Chen H X, Zhao Y and Hu J C 2009 Global earthquakes and volcanoes: Distribution and variations; Seismol. Geol. 31(4) 573-583 (in Chinese with English abstract).

Hou G T, Wang C C, Li J H and Qian X L 2006 Late Paleoproterozoic extension and a palaeostress field reconstruction of North China Craton; Tectonophys. 422 89-98.

Hussein H M, Abou Elenean K M, Marzouk I A, Korrat I M, Abu El-Nader I F, Ghazala $\mathrm{H}$ and ElGabry M N 2013 Present-day tectonic stress regime in Egypt and surrounding area based on inversion of earthquake focal mechanisms; J. Afr. Earth Sci. 81 1-15.

$\mathrm{Ju}$ W and Sun W F 2016 Tectonic fractures in the Lower Cretaceous Xiagou Formation of Qingxi Oilfield, Jiuxi Basin, NW China. Part two: Numerical simulation of tectonic stress field and prediction of tectonic fractures; J. Petrol. Sci. Eng. 146 626-636.

Ju W, Hou G T, Li L and Xiao F F 2012 End Late Paleozoic tectonic stress field in the southern edge of Junggar Basin; Geosci. Front. 3(5) 707-715.

Ju W, Hou G T and Hari K R 2013a Mechanics of mafic dyke swarms in the Deccan Large Igneous Province: Palaeostress field modelling; J. Geodyn. 66 79-91.

Ju W, Hou G T, Huang S Y and Ren K X 2013b Structural fracture distribution and prediction of the Lower Jurassic Ahe Formation sandstone in the Yinan-Tuzi area, Kuqa Depression; Geotecton. Metallog. 37(4) 592-602 (in Chinese with English abstract).

Ju W, Hou G T, Feng S B, Zhao W T, Zhang J Z, You Y, Zhan $\mathrm{Y}$ and $\mathrm{Yu} \mathrm{X}$ 2014a Quantitative prediction of the Yanchang Formation Chang $6_{3}$ reservoir tectonic fracture in the Qingcheng-Heshui area, Ordos Basin; Earth Sci. Front. 21(6) 310-320 (in Chinese with English abstract).

Ju W, Hou G T and Zhang B 2014b Insights into the damage zones in fault-bend folds from geomechanical models and field data; Tectonophys. 610 182-194.

Ju W, Sun W F and Hou G T 2015 Insights into the tectonic fractures in the Yanchang Formation intetbedded sandstone-mudstone of the Ordos Basin based on core data and geomechanical models; Acta Geol. Sin. Engl. 89(6) 1986-1997.

Kreemer C, Holt W E and Haines A J 2003 An integrated global model of present-day plate motions and plate boundary deformation; Geophys. J. Int. 154 8-34.

Li S D 1983 Principle of Dynamic Geology, Geological Publishing House, Beijing, 359p (in Chinese).

Lithgow-Bertelloni C and Richards M A 1998 The dynamics of Cenozoic and Mesozoic plate motions; Rev. Geophys. 36(1) 27-78.

Lund B and Townend J 2007 Calculating horizontal stress orientations with full or partial knowledge of the tectonic stress tensor; Geophys. J. Int. 170(3) 1328-1335.

Lunina O V and Gladkov A S 2007 Late Cenozoic fault pattern and stress fields in Barguzin rift (Baikal region); Russ. Geol. Geophys. 48 598-609.

Ma L H, Han Y B and Yin Z Q 2007 Distribution characteristics of global significant earthquakes and possible connection between earthquakes and Earth's variable 
rotation rate; Astron. Res. Techn. 4(4) 406-411 (in Chinese with English abstract).

Martin P, Hunen J V, Parmam S and Davidson J 2008 Why does plate tectonics occur only on Earth? Phys. Edu. 43(2) 144-150.

Naimi-Ghassabian N, Khatib M, Nazari H and Heyhat M 2015 Present-day tectonic regime and stress patterns from the formal inversion of focal mechanism data, in the North of Central-East Iran Blocks; J. Afr. Earth Sci. 111113 126.

Reynolds S D, Coblentz D D and Hillis R R 2002 Tectonic forces controlling the regional intraplate stress field in continental Australia: Results from new finite-element modelling; J. Geophys. Res. 107(B7), doi: 10.1029/ 2001JB000408.

Smart K J, Ferrill D A and Morris A P 2009 Impact of interlayer slip on fracture prediction from geomechanical models of fault-related folds; AAPG Bull. 93(11) $1447-$ 1458.

Soumaya A, Ayed N B, Delvaux D and Ghanmi M 2015 Spatial variation of present-day stress field and tectonic regime in Tunisia and surroundings from formal inversion of focal mechanisms: Geodynamic implications for central Mediterranean; Tectonics 34 1154-1180.

Sperner B, Muller B, Heidbach O, Delvaux D, Reinecker J and Fuchs K 2003 Tectonic stress in the Earth's crust: Advances in the World Stress Map Project; In: New Insights into Structural Interpretation and Modeling (ed.) Nieuwland D A, Geol. Soc. London, Spec. Publ. 212 101116.

Tang Y J 1997 A preliminary analysis on the character of the distribution of global earthquakes in latitude and the peak abnomaly near latitude $35^{\circ} \mathrm{N}$; North China Earthq. Sci. 15(2) 76-80 (in Chinese with English abstract).

Tingay M, Morley C, King R, Hillis R, Coblentz D and Hall R 2010 Present-day stress field of Southeast Asia; Tectonophys. 482 92-104.

Yu Y, Hong H J, Liu, P X, Tao W and Zheng X Z 2003 Spatio-temporal distribution of global great earthquakes and dynamic mechanism; Earth Sci. Front. 10 5-10 (in Chinese with English abstract).

Zoback M L 1992 First- and second-order patterns of stress in the lithosphere: The World Stress Map Project; J. Geophys. Res. 97(B8) 11,703-11,728.

Corresponding editor: N PURNACHANDRA RAO 